\title{
Incomplete Regulation, Asymmetric Information, and Collusion-Proofness
}

\author{
Marco Meireles and Paula Sarmento \\ Faculdade de Economia do Porto, Rua Dr. Roberto Frias, 4200-464 Porto, Portugal \\ Correspondence should be addressed to Marco Meireles, 070421008@fep.up.pt \\ Received 27 May 2011; Revised 2 October 2011; Accepted 3 October 2011 \\ Academic Editor: David E. Giles
}

Copyright (C 2012 M. Meireles and P. Sarmento. This is an open access article distributed under the Creative Commons Attribution License, which permits unrestricted use, distribution, and reproduction in any medium, provided the original work is properly cited.

In an incomplete regulation framework, the regulator cannot replicate all the possible outcomes by himself since he has no influence over some firms in the market. Due to asymmetric information, it may be better for the regulator to allow the unregulated firms to extract a truthful report from the regulated firm through side-payments under collusion, and therefore the "collusionproofness principle" may not hold. In fact, by introducing an exogenous number of unregulated firms, social welfare differences seem to favour a collusion-allowing equilibrium. However, such result will depend on the relative importance given by the regulator to the consumer surplus in the social welfare function.

\section{Introduction}

This paper aims to evaluate the optimal incomplete regulation contract when it is possible to form a coalition between unregulated firms and the regulated firm, and the marginal costs of the regulated firm are private information, not available to the regulator and neither to the other competitors. We will also evaluate how such results may be influenced by the relative importance given by the regulator to the consumer surplus in the social welfare function.

Most of the literature on economic regulation studied optimal contracts in the context of complete regulation, either between a regulator and a firm (monopoly) or between a regulator and all the firms in the market (usually a duopoly).

Baron and Myerson [1], Laffont and Tirole [2], and Lewis and Sappington [3] focused on the design of an optimal regulation contract in a monopoly under asymmetric information. On the first two papers the regulator was not aware of the monopolist's marginal costs, while in the last that private information was related to the market's demand function. They all concluded that the regulator has to pay a price above the marginal cost to avoid an untruthful report by the monopolist. Lewis and Sappington [3], however, also stated that under some conditions (nondecreasing marginal costs), and in spite of the private information about the demand function, the same first-best optimum equilibrium may be achieved.

Caillaud [4], Laffont and Martimort [5, 6], and Tangerås [7] extended optimal complete regulation under asymmetric information to a competition framework. Caillaud [4] focused on the informational effect that the existence of a competitive fringe had on the regulation of a dominant firm under asymmetric information. He concluded that the presence of a fringe is always welfare enhancing but its magnitude depends on the degree of information correlation and on the characteristics of the demand function. Both Laffont and Martimort [5, 6] and Tangerås [7] introduced the possibility of collusion between two firms in a context of complete regulation under asymmetric information. The first mainly emphasises the role of correlated information between the firms as a determinant of the strength of the coalition, concluding that in the presence of correlated private information the regulator can create a regulation contract which can replicate exactly the collusion outcome: the collusionproofness principle. The second paper studied the incentives for collusion when a market is regulated through yardstick competition. Since firms decided to collude before knowing their own productivity, the collusion would be costly to 
society only if firms could commit to the side payments agreed.

Still within the framework of complete regulation and asymmetric information, some literature focused on the effects of collusion on social welfare and analysed its potential benefits. While Che [8] studied a possible collusion between a regulator and a firm and how it might increase the regulator's monitoring effort, Itoh [9], Lawarrëe and Shin [10], and Shin [11] analysed the possibility of collusion between agents, through side-payments. They all have concluded that by allowing side-payments between agents, the regulator is able to more easily induce truthful reports from the firms, reducing the informational rent.

Biglaiser and Ma [12] and Aubert and Pouyet [13] are two very important contributions to the study of optimal regulation when the regulator is only able to make a contract with a dominant firm (incomplete regulation) under asymmetric information. Biglaiser and $\mathrm{Ma}$ [12] focus on optimal incomplete regulation when only the dominant firm has private information regarding the demand function and the unregulated competitor has some market power, acting as a Stackelberg follower. They proved that depending on the weight given by the regulator to consumer surplus on social welfare, the equilibrium outcome could be both separating and pooling or just separating. The Aubert and Pouyet's [13] model is the closest to the one we propose. They have worked in a framework of incomplete regulation under asymmetric information and with the possibility of collusion between the two firms operating in the market. Due to product imperfect substitutability, the unregulated competitor has incentives to bribe the regulated firm such that it overstates her costs and produces less. They have concluded that it is not optimal to design a collusion-proof contract for regulation as it imposes both distortions at the bottom and at the top (inefficient and efficient regulated firms, resp.), while by allowing collusion the regulator may induce the nonregulated firm to indirectly tax its competitor. The Collusion-Proofness Principle will not hold in the incomplete regulation framework since the regulator is unable to contract with the unregulated firm, and therefore it has limited possibilities for contracts when compared to what can be achieved within the coalition of the two firms. The main difference between the Collusion-Proof contract and a contract that allows collusion is that in the first the regulator has to pay larger amounts to the regulated firm to ensure that it reveals its efficiency despite collusion while in the last, the regulator uses collusion to make the unregulated firm pay to ensure that the regulated firm reveals its efficiency.

This paper's main contribution is to study the role of collusion in the optimal design of incomplete regulation under asymmetric information in the presence of more than one unregulated firms. The market is composed of a dominant (regulated) firm and by a small number of unregulated firm, which produce the same homogeneous product (although different from the one produced by the regulated firm) and choose their quantities as Stackelberg followers. Similarly to Aubert and Pouyet [13], we assume that the only private information on the market is the marginal cost of the dominant firm, which can take one of two possible values.
Additionally, we introduce differentiation among unregulated firms by admitting different marginal costs, which are publicly known. During entire the analysis we also use a similar social welfare function to the one used by Aubert and Pouyet [13], which allows us to compare the results directly. However, these assumptions may also be considered shortcomings and a reason for further research: generally the costs for the unregulated firms are also unknown and regulation is usually made in terms of price-cap, instead of quantities. As later showed in the paper, conclusions will also change dramatically by giving more weight to the consumer surplus in the social welfare function, emphasising the importance of the regulator's priorities when ensuring competition in a market.

This paper also has a wide range of empirical applicability. An example is the Portuguese fixed telecommunications market, which is constituted by a dominant regulated firm (Portugal Telecom) and by a small number of unregulated competitors. Once again, consumers view telecommunications services of Portugal Telecom as different from the other competitors. We may also apply the model to other sectors as Health and Education in Portugal. In each region, we could look at the health-care market as being composed of one (or two) dominant public (regulated) hospitals and by a small number of private hospitals that compete with most types of health services. The same can be said about the regional market for undergraduate degrees. Usually the public university is the dominant firm, regulated in terms of tuitions and there is also a small number of private universities that are free to impose the tuition they would like.

The paper is organized as follows. In the next section we describe the model and its characteristics, also describing the timing to better understand the sequence of events. In later sections we analyse the optimal regulation outcome (quantities, prices, profits, transfer from the regulator, sidepayment from the unregulated firms to the regulated firm and welfare level) in different frameworks within incomplete regulation: complete information, incomplete information in the absence of collusion and incomplete information with the possibility of collusion. Then we compare the outcome from contracts that are Collusion-Proof with the outcome obtained with contracts that allow collusion and assess which one entails a higher level of social welfare. Finally, we discuss the importance of the social welfare function to the robustness of such findings, by comparing the consumer surplus values in collusion-proofness and collusion-allowing equilibriums.

\section{The Model}

2.1. The Firms. The market is composed of a dominant regulated firm $F^{A}$ and by $n$ unregulated firms, each denoted by $F_{i}^{B}$, where $i=1, \ldots, n$. All the firms compete in quantities. Firm $F^{A}$ has a constant marginal cost $\theta^{a}$ which can take one of two possible values, $\underline{\theta}$ or $\bar{\theta}, \bar{\theta}-\underline{\theta} \equiv \Delta \theta>0$. The value of this marginal cost is private information for $F^{A}$; however, its distribution is public knowledge: with probability $\underline{p} F^{A}$, it is efficient $\left(\theta^{a}=\underline{\theta}\right)$ and it is inefficient $\left(\theta^{a}=\bar{\theta}\right)$ 
with probability $\bar{p}=1-\underline{p}$. The unregulated firms have different marginal costs $\left(\theta_{i}^{b}\right)$, which are publicly known. For simplification, we have assumed that the unregulated firms are ordered from the most efficient to the least efficient, such that $\theta_{1}^{b} \leq \theta_{2}^{b} \leq \ldots \leq \theta_{n}^{b}$.

2.2. The Consumers. The consumers have two differentiated products available for consumption: $q^{a}$ is the quantity of the product produced by the regulated firm in market $\mathrm{A}$ and $q_{i}^{b}$ is the quantity of the product produced by the unregulated firm $i$, where $Q^{b}=\sum_{i=1}^{n} q_{i}^{b}$ represents the total quantity produced by all unregulated firms. The Gross Consumer Surplus is given by

$$
G S\left(q^{a}, Q^{b}\right)=d^{a} q^{a}+d^{b} Q^{b}-\frac{1}{2}\left(q^{a}\right)^{2}-\frac{1}{2}\left(Q^{b}\right)^{2}-s q^{a} Q^{b},
$$

where the parameter $s \in(0,1)$ measures the degree of substitutability between the two products, whereas the parameter $d^{j}$ represents the size of market $j=A, B$ (Such Consumer Surplus function is a result from the standard quadratic utility function proposed by Dixit.) The inverse demand functions for both markets are given by

$$
\begin{aligned}
& P^{a}\left(q^{a}, Q^{b}\right)=d^{a}-q^{a}-s Q^{b}, \\
& P^{b}\left(q^{a}, Q^{b}\right)=d^{b}-Q^{b}-s q^{a} .
\end{aligned}
$$

For the time being, we assume that $d^{b}-d^{a}=\theta_{1}^{b}-\underline{\theta}=$ $\theta_{n}^{b}-\bar{\theta}$, which means that the difference between the marginal costs of the most efficient unregulated firm $\left(F_{1}^{b}\right)$ and the efficient type of $F^{A}$ is equal to the difference between the cost of the most inefficient firm in market $\mathrm{B}\left(F_{n}^{b}\right)$ and the inefficient type of the regulated firm, and they are both equal to the difference between the size of the two markets. (We will see later on that the conclusions remain the same if we disregard such assumption.)

2.3. The Regulator. Only firm $F^{A}$ is regulated by the regulator. The regulation contract is composed by a quantitytransfer pair $\left\{q^{a}\left(\theta_{m}^{a}\right), t^{a}\left(\theta_{m}^{a}\right)\right\}_{\theta_{m}^{a} \in\{\theta, \bar{\theta}\}}$, which depends on the message sent by the regulated firm about its cost to the regulator $\left(\theta_{m}^{a}\right)$. For a given contract $\left\{q^{a}, t^{a}\right\}$, the total expost profits are equal to

$$
\begin{gathered}
\pi^{a}=\left[P^{a}\left(q^{a}, Q^{b}\right)-\theta^{a}\right] q^{a}-t^{a}, \\
\pi_{i}^{b}=\left[P^{b}\left(q^{a}, Q^{b}\right)-\theta_{i}^{b}\right] q_{i}^{b} \quad \forall i=1, \ldots, n .
\end{gathered}
$$

Similarly to Aubert and Pouyet [13], we have decided not to consider any relationship between the regulator and any other firm. However, that could be introduced into the model by extending the analysis to a lump-sum tax or taxes proportional either to profits or to output.

We also assume that the firms' profits do not enter the objective function of the regulator, so that this objective is reduced to the net consumer surplus and to the transfers paid by the regulated firm. Therefore, the rents left to the regulated firm are socially costly since they represent the informational rent paid by the regulator to induce a truthful report from the regulated firm. The regulator's objective is to maximize social welfare, given by

$$
W=G S\left(q^{a}, Q^{b}\right)-\theta^{a} q^{a}-P^{b}\left(q^{a}, Q^{b}\right) Q^{b}-\pi^{a} .
$$

2.4. The Timing. In this model we have a sequential game, described as follows.

(1) Nature draws one of the two possible values for $\theta^{a}$, only known to the regulated firm $F^{A}$.

(2) The regulator proposes a contract $\left\{q^{a}\left(\theta_{m}^{a}\right)\right.$, $\left.t^{a}\left(\theta_{m}^{a}\right)\right\}_{\theta_{m}^{a} \in\{\theta, \bar{\theta}\}}$ to the regulated firm $F^{A}$, without knowing her marginal costs.

(3) The regulated firm $F^{A}$ decides whether to accept or reject this contract. If she refuses, she gets a reservation gain exogenously normalized to zero and the game stops. If $F^{A}$ accepts the contract, the game continues.

(4) After accepting the contract and before the report is sent to the regulator, the unregulated firms may try to pay $F^{A}$ the amount $b$ in order to induce her to always claim to be inefficient to the regulator: collusion. After the collusion agreement takes place, $F^{A}$ sends a message $\theta_{m}^{a}$ to the regulator, produces the corresponding quantity, and receives the transfer accordingly.

(5) All the unregulated firms act as Stackelberg followers, deciding their individual quantities simultaneously, given the quantity produced by $F^{A}$.

\section{Optimal Regulation with Perfect Information}

In this section we assume that all the firms' marginal costs are known by all. The best response function for each of the unregulated firms is given by

$$
q_{i}^{b}=\frac{d^{b}-\theta_{i}^{b}-s q^{a}-\sum_{j \neq i} q_{j}^{b}}{2}, \quad \forall i=1, \ldots, n .
$$

Since all unregulated firms decide their quantities simultaneously, it is possible to consider an aggregate best response function for market $\mathrm{B}$, given by

$$
Q^{b}=\frac{1}{n+1}\left[n d^{b}-\sum_{i=1}^{n} \theta_{i}^{b}-n s q^{a}\right] .
$$

3.1. Complete Regulation and Complete Information: The Social Optimum. The socially optimal quantities $\left(q_{\mathrm{opt}}^{a}, q_{\mathrm{iop}}^{b}\right.$, $Q_{\text {opt }}^{b}$ ) would be the ones that the regulator would ideally choose if he had complete information and complete regulation, becoming a reference benchmark to other possible situations. These optimal quantities are such that the prices equal the marginal costs (for the firms operating in market $\mathrm{B}$, the optimal quantities are set by setting the price equal to the marginal cost of the least efficient firm (firm $n$ ). Hence, 
we are implicitly considering an exogenous process of entry and exit of firms in this market) and are given by

$$
\begin{aligned}
q_{\mathrm{opt}}^{a}= & \frac{d^{a}-\theta^{a}-s\left(d^{b}-\theta_{n}^{b}\right)}{1-s^{2}}, \\
q_{\mathrm{iopt}}^{b}=\theta_{n}^{b}-\theta_{i}^{b} & \frac{d^{b}-\theta_{n}^{b}-s\left(d^{a}-\theta^{a}\right)}{1-s^{2}} .
\end{aligned}
$$

Notice that the only two relevant marginal costs for the optimal quantities are the ones related to $F^{A}$ and $F_{n}^{B}$ since they are directly linked to the equilibrium prices for both markets. Such quantities also depend positively on the market dimension and negatively on the competitor's market dimension. It is also worth mentioning that none of the firstbest equilibrium prices depend on the number of firms in market $\mathrm{B}(n)$. Another interesting feature is that the quantity produced by each of the unregulated firms is directly given by the absolute costs advantage, leading to a quantity equal to zero for the least efficient firm.

3.2. Incomplete Regulation and Complete Information: The Second Best. In this section we assume that the regulator is only able to regulate one firm $\left(F^{A}\right)$, whose marginal costs' are perfectly observable. As the rent of $F^{A}$ is socially costly, the regulator will decide to extract all profits from the regulated firm. By incorporating this constraint into the social welfare function stated previously, we reach the following solution (see Appendix A. for the objective function, constraints and first order conditions), for any $\theta^{a}=\{\underline{\theta}, \bar{\theta}\}$ :

$$
\begin{aligned}
q_{*}^{a}\left(\theta^{a}\right)= & \frac{1}{(n+1)^{2}-(n s)^{2}} \\
& \times\left[(n+1)^{2}\left(d^{a}-\theta^{a}\right)-n s\left(n d^{b}-\sum_{i=1}^{n} \theta_{i}^{b}\right)\right], \\
q_{i *}^{b}\left(q_{*}^{a}\left(\theta^{a}\right)\right)= & \frac{1}{(n+1)^{2}-(n s)^{2}} \\
& \times\left[(n+1)\left(d^{b}-s\left(d^{a}-\theta^{a}\right)\right)\right. \\
& +\left(n+1-n s^{2}\right) \sum_{j \neq i} \theta_{j}^{b} \\
Q_{*}^{b}\left(q_{*}^{a}\left(\theta^{a}\right)\right)= & \frac{\left.+n\left(s^{2}(n-1)-(n+1)\right) \theta_{i}^{b}\right],}{(n+1)^{2}-(n s)^{2}} \\
& \times\left[n d^{b}-\sum_{i=1}^{n} \theta_{i}^{b}-n s\left(d^{a}-\theta^{a}\right)\right] .
\end{aligned}
$$

Notice that if $s=0$ the two quantities $q_{*}^{a}\left(\theta^{a}\right)$, are the same as the equilibrium quantities in Section 3.1., meaning that when the two products are independent, the unregulated firms do not compete with the regulated firm, and therefore there is no way that the regulator can influence their behaviour in an incomplete regulation framework. However, when the two products are substitutes, and as the profits of the unregulated firms are not included in the social welfare function, the regulator wants to incentive firm $F^{A}$ to produce more such that the Consumer Surplus is greater, even if that means smaller profits for the unregulated firms. Also notice that all optimal quantities depend on the number of firms in market B $(n)$.

\section{Optimal Incomplete Regulation under Asymmetric Information and in the Absence of Collusion}

We now assume that the regulator and all the unregulated firms do not know the firm $F^{A}$ 's actual marginal costs and only its distribution is publicly known. We also assume that the regulator can only regulate $F^{A}$ and the marginal costs of the unregulated firms are perfectly observable and that collusion cannot occur.

According to the timing defined previously, after $F^{A}$ decides which quantity to produce, the other firms will simultaneously choose how much they want to produce according to their respective best response functions.

We denote $\pi^{a}\left(\theta^{a}, \theta_{m}^{a}\right)$ as the profits for $F^{A}$ when she has a marginal cost of $\theta^{a}$ and reports to have a marginal cost of $\theta_{m}^{a}$ to the regulator, $\overline{q^{a}} \equiv q^{a}\left(\overline{\theta^{a}}\right)$ as the quantity that should be produced by the regulated firm when inefficient, and $q^{a} \equiv q^{a}\left(\underline{\theta^{a}}\right)$ as the quantity produced when $F^{A}$ is efficient. Even in the presence of incomplete regulation, we can still assume that the revelation principle holds (see Green and Laffont [14] or Myerson [15], among others, on the revelation principle), and we can focus on direct and truthful contracts, especially on $\underline{\pi}^{a} \equiv \pi^{a}\left(\underline{\theta}^{a}, \underline{\theta^{a}}\right)$ and $\overline{\pi^{a}} \equiv \pi^{a}\left(\overline{\theta^{a}}, \overline{\theta^{a}}\right)$ which represent the rents for the efficient and inefficient regulated firms at a truthful equilibrium, respectively. Let us also denote $\overline{Q^{b}}$ and $Q^{b}$ as the total quantity produced in market B when $F^{A}$ is inefficient and efficient, respectively.

Therefore, the regulator's objective is to maximize social welfare, subject to incentive compatibility (ICC) and participation constraints (PC), as follows:

$$
\begin{aligned}
& \max _{\left\{\underline{a}, \bar{q}^{a}\right\}} E(W)=\underline{p}\left\{d^{a} \underline{q}^{a}+d^{b} \underline{Q^{b}}-\frac{1}{2}\left(\underline{q^{a}}\right)^{2}-\frac{1}{2}\left(\underline{Q^{b}}\right)^{2}-s \underline{q^{a}} \underline{Q^{b}}\right. \\
& \left.-\underline{\theta} q^{a}-\left(d^{b}-\underline{Q^{b}}-s \underline{q^{a}}\right) \underline{Q^{b}}-\underline{\pi^{a}}\right\} \\
& +\bar{p}\left\{d^{a} \overline{q^{a}}+d^{b} \overline{Q^{b}}-\frac{1}{2}\left(\overline{q^{a}}\right)^{2}-\frac{1}{2}\left(\overline{Q^{b}}\right)^{2}-s \overline{q^{a}} \overline{Q^{b}}\right. \\
& \left.-\bar{\theta} \overline{q^{a}}-\left(d^{b}-\overline{Q^{b}}-s \overline{q^{a}}\right) \underline{Q^{b}}-\overline{\pi^{a}}\right\},
\end{aligned}
$$

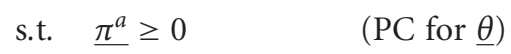

$$
\begin{aligned}
& \overline{\pi^{a}} \geq 0 \quad(\mathrm{PC} \text { for } \bar{\theta}) \\
& \underline{\pi^{a}} \geq \overline{\pi^{a}}+\Delta \theta \overline{q^{a}} \quad(\text { ICC for } \underline{\theta}) \\
& \overline{\pi^{a}} \geq \underline{\pi^{a}}-\Delta \theta \underline{q^{a}} \quad(\text { ICC for } \bar{\theta}) \text {. }
\end{aligned}
$$

As usual, only the PC for the inefficient $F^{A}$ and the ICC for the efficient regulated firm constraints are binding. 
Substituting these constraints into the objective function and using the first-order conditions, we get

$$
\begin{aligned}
\underline{q^{a}}= & \frac{1}{(n+1)^{2}-(n s)^{2}} \\
& \times\left[(n+1)^{2}\left(d^{a}-\underline{\theta}\right)-n s\left(n d^{b}-\sum_{i=1}^{n} \theta_{i}^{b}\right)\right]=q_{*}^{a}(\underline{\theta}) \\
\overline{q^{a}}= & \frac{1}{(n+1)^{2}-(n s)^{2}} \\
& \times\left[(n+1)^{2}\left(d^{a}-\bar{\theta}\right)-n s\left(n d^{b}-\sum_{i=1}^{n} \theta_{i}^{b}\right)\right] \\
& -\frac{(n+1)^{2}}{(n+1)^{2}-(n s)^{2}} \frac{p}{\bar{p}} \Delta \theta=q_{*}^{a}(\bar{\theta}) \\
& -\frac{(n+1)^{2}}{(n+1)^{2}-(n s)^{2}} \frac{p}{\bar{p}} \Delta \theta .
\end{aligned}
$$

Under asymmetric information and incomplete regulation and in the case where firms cannot collude we can verify that the standard no distortion at the top equilibrium in an adverse selection model holds. An efficient firm $F^{A}$ will produce the complete information output; however, the regulator needs to leave an information rent of $\Delta \theta \overline{q^{a}}$ in order to induce a truthful revelation of its efficiency. Since this rent increases with the quantity produced by the inefficient firm $F^{A}$, this quantity will be distorted downwards relatively to the respective complete information quantity. This informational rent also increases with the probability of firm $F^{A}$ being efficient $(p)$ and with the substitutability degree between the products $\mathrm{A}$ and $\mathrm{B}$. The decrease in the quantity for an inefficient regulated firm does not have large consequences on the social welfare, since the risk of having an inefficient $F^{A}$ is lower and consumers can more easily compensate this decrease in $\overline{q^{a}}$ by consuming more from the other unregulated firms. The informational rent will also increase with the number of unregulated firms $n$. Once again, a lower $\overline{q^{a}}$ will lead to smaller distortions on the expected social welfare since the unregulated firms' ability of capturing higher profits will be very low when they are many.

At this point we should also notice that due to product substitutability there is a stake for collusion (Proof is in Appendix B). We have seen that the ICC for the efficient $F^{A}$ will lead to $q^{a} \geq \overline{q^{a}}$, also leading to $\overline{\pi_{i}^{b}} \geq \pi_{i}^{b}, \forall i=1, \ldots, n$, and therefore the unregulated firms have incentives to make the regulated firm always report to be inefficient. Since these unregulated firms' profits increase will play an important role in the next section, we will denote $\Delta \pi_{i}^{b} \equiv \overline{\pi_{i}^{b}}-\pi_{i}^{b}$ as the increase in the profits of firm $i$ in the unregulated sector when $F^{A}$ produces $\overline{q^{a}}$ instead of $\underline{q^{a}}$.

\section{Optimal Regulation under Asymmetric Information and with the Possibility of Collusion}

As it was previously mentioned, it is possible for the firms to collude such that the firm $F^{A}$ always reports to be inefficient to the regulator. Such decision takes place after the firm $F^{A}$ knows her true costs but before deciding which costs to report to the regulator. To model collusion under asymmetric information we used a methodology similar to that of Laffont and Martimort [5, 6] and Aubert and Pouyet [13]. Since $\theta^{a}$ is private information at the time when the collusion takes place, we can model the bargaining process between the firms by considering a hypothetical benevolent mediator whose objective is to maximize the collusion's aggregate expected profits, subject to participation and incentive compatibility constraints. This mediator can be viewed as another principal to whom the revelation principle applies: the mediator will offer a collusive agreement such that all firms are willing to participate and firm $F^{A}$ truthfully reports its type to the coalition. We should also notice that since the marginal costs of all the unregulated firms are publicly known, they cannot deviate from their best response function to a certain report from the firm $F^{A}$, leading to an immediate detection of the collusion by an antitrust authority. Conversely, this authority does not detect any type of collusion between the firms if they act according to the report made by $F^{A}$ to the regulator.

The aim of this section is to analyse to what extent the collusion-proofness principle stated by Laffont and Martimort $[5,6]$ holds in the present framework. With complete regulation, there is no loss of generality if we focus only on regulation contracts that will replicate the collusion outcomes since the regulator is able to enforce any output to all firms in the market. However, as already pointed out by Aubert and Pouyet [13], in an incomplete regulation setting, the regulator is not able to replicate all possible outcomes using a regulation contract, and therefore we should not restrict our attention only to those contracts that avoid collusion. All situations that involve a side-payment from the unregulated firms to the regulated firm $F^{A}$ are out of the regulator's reach since he cannot impose any tax on any firm $F_{i}^{B}$ which could replicate those payments. By comparing the social welfare from Collusion-Proofness Contracts with the social welfare obtained through contracts that allow collusion, we are able to assess the robustness of the Collusion-Proof Principle.

5.1. Collusion-Proof Contracts. We start by focusing on Collusion-Proof Contracts, which are contracts that induce a passive response from the coalition. When designing such a contract, the mediator will ask firm $F^{A}$ to truthfully report its marginal costs to the regulator and no side-payment will be made from the unregulated firms to $F^{A}$.

Let us denote $\theta_{m}^{a}\left(\theta^{a}\right)$ as the report recommended by the mediator when the marginal cost of firm $F^{A}$ is $\theta^{a}$, and let $\theta^{n c}\left(\theta^{a}\right)$ be the report that firm $F^{A}$ would make to the regulator if no collusion occurs. In the case of collusion, the regulated firm will produce $q^{a}\left(\theta_{m}^{a}\right)$, and each of the unregulated firms would have to produce $q_{i}^{b}\left(\theta_{m}^{a}\right)$ and pay firm $F^{A}$ the amount $b_{i}\left(\theta^{a}\right)$, where $B\left(\theta^{a}\right)=\sum_{i=1}^{n} b_{i}\left(\theta^{a}\right)$ represents the aggregate side-payment made to firm $F^{A}$.

The mediator's objective is to maximize the coalition's aggregate profits subject to the participation constraints (PCs) for all firms and to the incentive compatibility constraints (ICCs) for both types of the regulated firm such 
that it will always reveal truthfully its marginal cost to the coalition. It can be written as

$$
\begin{aligned}
& \max _{\left\{\left(\theta_{m}^{a}\right), b_{i}\left(\theta^{a}\right)\right\}_{i=1}^{n}} E_{\theta^{a}}\left[\sum_{i=1}^{n} \pi_{i}^{b}\left(\theta_{m}^{a}\right)+\pi^{a}\left(\theta_{m}^{a}, \theta^{a}\right)\right] \\
& \text { s.t. } \pi^{a}\left(\theta_{m}^{a}(\underline{\theta}), \underline{\theta}\right)+B(\underline{\theta}) \geq \pi^{a}\left(\theta^{n c}(\underline{\theta}), \underline{\theta}\right) \text {, } \\
& \text { PC, for } F^{a} \\
& \pi^{a}\left(\theta_{m}^{a}(\bar{\theta}), \bar{\theta}\right)+B(\bar{\theta}) \geq \pi^{a}\left(\theta^{n c}(\bar{\theta}), \bar{\theta}\right), \\
& \text { PC for } F^{a} \\
& \pi^{a}\left(\theta_{m}^{a}(\underline{\theta}), \underline{\theta}\right)+B(\underline{\theta}) \geq \pi^{a}\left(\theta_{m}^{a}(\bar{\theta}), \underline{\theta}\right)+B(\bar{\theta}), \\
& \text { ICC for } F^{a} \\
& \pi^{a}\left(\theta_{m}^{a}(\bar{\theta}), \bar{\theta}\right)+B(\bar{\theta}) \geq \pi^{a}\left(\theta_{m}^{a}(\underline{\theta}), \bar{\theta}\right)+B(\underline{\theta}), \\
& \text { ICC for } F^{a} \\
& \pi_{i}^{b}\left(\theta_{m}^{a}(\underline{\theta})\right)-b_{i}(\underline{\theta}) \geq \pi_{i}^{b}\left(\theta^{n c}(\underline{\theta})\right), \\
& \forall i=1, \ldots, n, \quad \text { PC for } F_{i}^{b} \\
& \pi_{i}^{b}\left(\theta_{m}^{a}(\bar{\theta})\right)-b_{i}(\bar{\theta}) \geq \pi_{i}^{b}\left(\theta^{n c}(\bar{\theta})\right), \\
& \forall i=1, \ldots, n, \quad \text { PC for } F_{i}^{b} .
\end{aligned}
$$

It is possible to prove that in the case of a CollusionProof contract, the regulator is able to use the information asymmetry within the coalition to create a regulation contract which leads to "individual" truthful revelation by firm $F^{A}$. Hence, the ICC for the inefficient type will not be binding, and the collusive equilibrium would be the same as in complete information (See proof in Appendix C). This result is opposite to the one found by Laffont and Martimort [6] in a complete regulation context, where asymmetric information generated inefficiencies in the functioning of the coalition.

By solving the mediator's problem, we get the CollusionProofness Constraint which the regulator needs to include in his problem in order to induce a passive response from the coalition. This constraint can be written as (see proof in Appendix C):

$$
\underline{\pi^{a}} \geq \overline{\pi^{a}}+\Delta \theta \overline{q^{a}}+\sum_{i=1}^{n} \Delta \pi_{i}^{b} .
$$

This result is consistent with the one reached by Aubert and Pouyet [13] for the case of two firms. Such constraint can be understood intuitively: an efficient firm $F^{A}$ is willing to report truthfully its marginal costs whenever its profits from telling the truth are greater than the ones she would get by pretending to be inefficient plus the highest amount of sidepayment that the unregulated firms are willing to give to firm $F^{A}$ (which is equal to the increase on their profits when the regulated firms mislead the regulator).
By solving the regulator's problem and including the collusion-proofness constraint derived above, the best separating collusion-proof contract is characterized by the following rents and quantities (see proof in Appendix C):

$$
\begin{aligned}
\underline{q_{c p}^{a}}= & \frac{1}{(n+1)^{2}-n s^{2}(2+n)} \\
& \times\left[(n+1)^{2}\left(d^{a}-\underline{\theta}\right)-(2+n) s\left(n d^{b}-\sum_{i=1}^{n} \theta_{i}^{b}\right)\right], \\
\overline{q_{c p}^{a}}= & \frac{1}{(n+1)^{2}-n s^{2}\left(n-2 \frac{\overline{\bar{p}}}{\bar{p}}\right)} \\
& \times\left[(n+1)^{2}\left(d^{a}-\bar{\theta}-\frac{\bar{p}}{\bar{p}} \Delta \theta\right)-s\left(n-2 \frac{\overline{\underline{p}}}{\bar{p}}\right)\right. \\
& \left.\times\left(n d^{b}-\sum_{i=1}^{n} \theta_{i}^{b}\right)\right],
\end{aligned}
$$

$\overline{\pi_{c p}^{a}}=0$

$\underline{\pi_{c p}^{a}}=\Delta \theta \overline{q_{c p}^{a}}+\sum_{i=1}^{n} \Delta \pi_{i}^{b}$.

Notice that both quantities are distorted when compared with the complete information outcomes and that this best collusion-proof contract is separating only if $q_{c p}^{a} \geq \overline{q_{c p}^{a}}$. Otherwise it entails pooling for a quantity given by (see proof in Appendix C):

$$
\begin{aligned}
q_{\text {pool }}^{a}= & \frac{1}{(n+1)^{2}-(n s)^{2}} \\
& \times\left[(n+1)^{2}\left(d^{a}-\bar{\theta}\right)-n s\left(n d^{b}-\sum_{i=1}^{n} \theta_{i}^{b}\right)\right],
\end{aligned}
$$

which is the complete information outcome for an inefficient $F^{A}$.

With collusion-proof contracts, the regulator has to provide $F^{A}$ with an extra rent in order to compensate for any side-payment that she might get through collusion. Since such amount is affected by the quantity produced by both an efficient and an inefficient firm $F^{A}$, they will be distorted downwards to minimize the cost of inducing truthful revelation, contrary to the standard result of no distortion at the top. In fact, to ensure collusion-proofness, the regulator has to reduce simultaneously the rent $\Delta \theta \overline{q_{c p}^{a}}$ and the stake for collusion $\sum_{i=1}^{n} \Delta \pi_{i}^{b}$. The first implies a decrease in $\overline{q_{c p}^{a}}$ while the second requires a simultaneous decrease in $q_{c p}^{a}$ and an increase of $\overline{q_{c p}^{a}}$ to bring these two quantities closer together. We have thus two opposite effects on $\overline{q_{c p}^{a}}$. As in Aubert and Pouyet [13], we can verify that such quantity will be smaller than in the case with no possibility of collusion, meaning that the first effect dominates the second.

The second objective of bringing both quantities closer together also conflicts with the condition of sustainability of a separating equilibrium for collusion-proof contracts: $q_{c p}^{a} \geq \overline{q_{c p}^{a}}$. Taken to an extreme, a pooling equilibrium 
may emerge. Such equilibrium will be especially desirable when the stake of collusion $\sum_{i=1}^{n} \Delta \pi_{i}^{b}$ is very large and very sensitive to the regulated quantities. This occurs when the profitability of the unregulated market is high $\left(n d^{b}-\right.$ $\left.\sum_{i=1}^{n} \theta_{i}^{b}\right)$ and when $F^{A} s$ scale of production $\left(q^{a}+\overline{q^{a}}\right)$ is low. Contrary to the findings of Aubert and Pouyet [13], the stake for collusion does not depend monotonically on the substitutability between the two products. It becomes larger for an increase in $s$ when the products are almost independent ( $s$ close to zero), and it also becomes larger for a decrease in $s$ for almost perfect substitutable products ( $s$ close to one). The stake for collusion is also larger for a small number of firms operating in the unregulated sector and decreases when $n$ increases.

5.2. Collusion-Allowing Contracts. As already mentioned, with incomplete regulation we cannot rule out contracts that allow collusion since there are some outcomes only attainable by taxing the unregulated firms and therefore unattainable by the regulator. A potential benefit of contracts inducing active collusion is precisely the possibility of extracting some rents from the unregulated firms and direct them to the regulated firm through side-payments, as also stated by Tirole [16], Itoh [9], Lawarrëe and Shin [10], and Shin [11]. Allowing collusion, the regulated firm $F^{A}$ will have higher utility and therefore will be more willing to accept the regulation contract even if it yields higher taxes.

As in the Aubert and Pouyet [13] model, it is possible to show that the revelation principle still applies to the present model framework, where the agent to be considered is the coalition as a whole and no longer the regulated (Proof is in Appendix D.) Hence, we can restrict our attention to direct mechanisms which induce active collusion and truthful reports from the coalition. The regulator must now include in his problem the incentive compatibility constraint and the participation constraint for the coalition, as well as the incentive compatibility constraints and participation constraints within the coalition, which will reflect the expected aggregate profits:

$$
\begin{aligned}
& \max _{\left\{\underline{\left.q^{a}, \overline{q^{a}}, b_{i}(\underline{\theta}), b_{i}(\bar{\theta})\right\}_{i=1}^{n}}\right.} E_{\theta^{a}}(W)=\underline{p}\left\{d^{a} \underline{q^{a}}+d^{b} \underline{Q^{b}}-\frac{1}{2}\left(\underline{q^{a}}\right)^{2}\right. \\
& -\frac{1}{2}\left(\underline{Q^{b}}\right)^{2}-s \underline{q^{a}} \underline{Q^{b}}-\underline{\theta} q^{a} \\
& \left.-\left(d^{b}-\underline{Q^{b}}-s \underline{q^{a}}\right) \underline{Q^{b}}-\underline{\pi^{a}}\right\} \\
& +\bar{p}\left\{d^{a} \overline{q^{a}}+d^{b} \overline{Q^{b}}-\frac{1}{2}\left(\overline{q^{a}}\right)^{2}\right. \\
& -\frac{1}{2}\left(\overline{Q^{b}}\right)^{2}-s \overline{q^{a}} \overline{Q^{b}}-\overline{\bar{\theta} q^{a}} \\
& \left.-\left(d^{b}-\overline{Q^{b}}-s \overline{q^{a}}\right) \underline{Q^{b}}-\overline{\pi^{a}}\right\} \\
& \text { s.t. } \quad \pi^{a}+\sum_{i=1}^{n} \underline{\pi_{i}^{b}} \geq \overline{\pi^{a}}+\Delta \theta \overline{q^{a}}+\sum_{i=1}^{n} \overline{\pi_{i}^{b}}
\end{aligned}
$$

ICC for coalition $(\underline{\theta})$

$$
\begin{aligned}
& \overline{\pi^{a}}+\sum_{i=1}^{n} \overline{\pi_{i}^{b}} \geq \underline{\pi^{a}}-\Delta \theta \underline{q^{a}}+\sum_{i=1}^{n} \underline{\pi_{i}^{b}} \\
& \text { ICC for coalition }(\bar{\theta}) \\
& \underline{\pi^{a}+\sum_{i=1}^{n} \underline{\pi_{i}^{b}} \geq 0}
\end{aligned}
$$

PC for coalition $(\underline{\theta})$

$$
\overline{\pi^{a}}+\sum_{i=1}^{n} \overline{\pi_{i}^{b}} \geq 0
$$

PC for coalition $(\bar{\theta})$

$$
\begin{aligned}
& \underline{\pi^{a}}+B(\underline{\theta}) \geq \pi^{a}\left(\theta^{n c}(\underline{\theta}), \underline{\theta}\right), \\
& \text { PC for } F^{a} \\
& \overline{\pi^{a}}+B(\bar{\theta}) \geq \pi^{a}\left(\theta^{n c}(\bar{\theta}), \bar{\theta}\right),
\end{aligned}
$$

PC for $F^{a}$

$$
\underline{\pi^{a}}+B(\underline{\theta}) \geq \pi^{a}\left(\theta_{m}^{a}(\bar{\theta}), \underline{\theta}\right)+B(\bar{\theta}),
$$

ICC for $F^{a}$

$$
\overline{\pi^{a}}+B(\bar{\theta}) \geq \pi^{a}\left(\theta_{m}^{a}(\underline{\theta}), \bar{\theta}\right)+B(\underline{\theta}),
$$

ICC for $F^{a}$

$$
\begin{array}{r}
\underline{\pi_{i}^{b}}-b_{i}(\underline{\theta}) \geq \pi_{i}^{b}\left(\theta^{n c}(\underline{\theta})\right), \quad \forall i=1, \ldots, n, \\
\text { PC for } F_{i}^{b} \\
\overline{\pi_{i}^{b}}-b_{i}(\bar{\theta}) \geq \pi_{i}^{b}\left(\theta^{n c}(\bar{\theta})\right), \quad \forall i=1, \ldots, n, . \\
\text { PC for } F_{i}^{b}
\end{array}
$$

Given the monotonicity condition that $q_{a c}^{a} \geq \overline{q_{a c}^{a}}$, the last six constraints will not be binding. Rearranging the other constraints it will yield the only two relevant conditions:

$$
\begin{gathered}
\underline{\pi^{a}}=\Delta \theta \overline{q^{a}}-\sum_{i=1}^{n} \underline{\pi_{i}^{b}}, \\
\overline{\pi^{a}}+\sum_{i=1}^{n} \overline{\pi_{i}^{b}}=0 .
\end{gathered}
$$

Hence, the best contract with active collusion is then characterized by the following rents and quantities:

$$
\begin{aligned}
\underline{q_{a c}^{a}}= & \frac{1}{\left(1-s^{2}\right) n(n+2)+1} \\
& \times\left[(n+1)^{2}\left(d^{a}-\underline{\theta}\right)-s(n+2)\left(n d^{b}-\sum_{i=1}^{n} \theta_{i}^{b}\right)\right]
\end{aligned}
$$




$$
\begin{aligned}
\overline{q_{a c}^{a}}= & \frac{1}{\left(1-s^{2}\right) n(n+2)+1} \\
\times & {\left[(n+1)^{2}\left(d^{a}-\bar{\theta}-\frac{p}{\bar{p}} \Delta \theta\right)\right.} \\
& \left.-s(n+2)\left(n d^{b}-\sum_{i=1}^{n} \theta_{i}^{b}\right)\right] \\
\frac{\pi_{a c}^{a}}{=} & \Delta \theta \overline{q_{a c}^{a}}-\sum_{i=1}^{n} \frac{\pi_{i}^{b}}{\overline{\pi_{a c}^{a}}=} \\
& -\sum_{i=1}^{n} \overline{\pi_{i}^{b}}
\end{aligned}
$$

By comparing these quantities with the ones obtained for the collusion-proof contract, we can verify that $q_{a c}^{a}=$ $q_{c p}^{a}$ and $\overline{q_{a c}^{a}}<\overline{q_{c p}^{a}}$. This can be intuitively understood. By allowing collusion, the regulator is aware that $F^{A}$ will have higher profits when she reports to be inefficient due to the side-payments made by the other firms. Hence, instead of rewarding efficiency, the regulator penalizes even further $F^{A}$ if she reports to be inefficient. In another way, when allowing active collusion, the regulator is now internalizing not only the benefit that the consumers derive from $q^{b}$ but also the profits that this consumption generates, which will be forwarded to the regulated firm through side-payments. Hence, imposing lower quantities for $F^{A}$ will increase the profits of the unregulated firms, and therefore smaller will be the share that the regulator needs to transfer to $F^{A}$.

5.3. Equilibrium Quantities Comparison. From this section onwards, due to algebraic complexity, we have decided to compare quantities, social welfare, and Consumer Surplus using graphical representations as a result of a computational simulation, using a set of parameters (See Appendix E. for parameter values used and detailed description of the simulation.) We will start by comparing $F^{A}$ 's and the unregulated firms' equilibrium quantities for both collusionproof and collusion-allowing contracts.

First, we will analyse how the equilibrium quantities produced by firm $F^{A}$ change with the number of competitors.

From the diagrams above. From Figure 1, we can see that there is a negative (and convex) relationship between the quantities produced by $F^{A}$ and the amount of competitors she faces in the unregulated market. Although these reflect a relatively low substitutability between the products, we can see that when the number of competitors remains low but increases (between 1 and 10), it has a dramatic effect on the quantity produced by $F^{A}$. The same happens for other values of the substitutability degree (as we will see later on), where the quantity of $F^{A}$ will tend to zero after a certain number of competitors.

The following graphs allow us to better understand how the two types of equilibrium quantities for $F^{A}$ and for all unregulated firms are related.

We can see that for an efficient $F^{A}$ it is indifferent to allow collusion or not, as expected. However, if $F^{A}$ is inefficient then we have different levels of output for the two types of contracts. Her quantities tend to be lower when collusion is allowed, which is an evidence that the competitors are able to detect firm $F^{A}$ 's inefficiency and that the truth-telling constraint holds. We should also notice that the total output produced in market B increases with the number of firms belonging to that market and that it is slightly higher in collusion-allowing contracts (Figure 2).

The following diagrams compare firm $F^{A}$ 's equilibrium outcomes for different levels of the substitutability degree.

We can immediately notice that when firm $F^{A}$ is efficient, her equilibrium quantities are approximately the same across the two possible situations, similarly to the result regarding the number of unregulated firms. However, when firm $F^{A}$ is inefficient the collusion-proof quantities are greater than the equilibrium quantities when collusion is allowed. Once more, this is evidence that when designing the collusive agreement, the unregulated firms are able to extract from $F^{A}$ truthful information about her costs (Figure 3 ).

The results obtained for increasing values of the substitutability degree are also very intuitive. If $F^{A}$ is efficient, her equilibrium quantities will increase as the consumers start to perceive the products as close substitutes. The opposite happens when $F^{A}$ is inefficient, where her quantities decrease with the degree of substitutability and become zero after a certain threshold.

It is also worth mentioning that the level of equilibrium quantities for $F^{A}$ also decrease when we increase the number of unregulated firms from two to five, and such pattern continues throughout the rest of the levels considered.

5.4. Social Welfare Differences. The goal of this section is to compare the levels of social welfare obtained by a collusionproof contract and by a contract that allows collusion. Let us start by looking at the differences between the value of expected Welfare when we change the number of unregulated firms (Figure 4).

It can be seen that the Welfare difference is always positive, meaning that the collusion-allowing equilibrium always entails a higher-expected Welfare than the one given by collusion-proof contracts. Furthermore, this value is increasing with the number of unregulated firms, and its level is slightly increasing with substitutability degrees. Such result can be justified in two ways. First, as we have argued before, the advantage of allowing collusion is that the unregulated firms in an effort to collude and decide side-payments will eventually lead firm $F^{A}$ to reveal its real costs, eliminating part of the information asymmetry. Such result would lead to a much higher cost for the regulator if collusion was not allowed. Secondly, as the number of unregulated firms increase the quantity and the relative weight of firm $F^{A}$ on the total output produced by both markets decrease, and therefore it will also decrease its importance in terms of social welfare.

Next, we will analyse the influence of the substitutability degree on Welfare differences, similarly to Aubert and Pouyet [13]. Additionally, we will consider four different values for the number of firms in market B (Figure 5). 

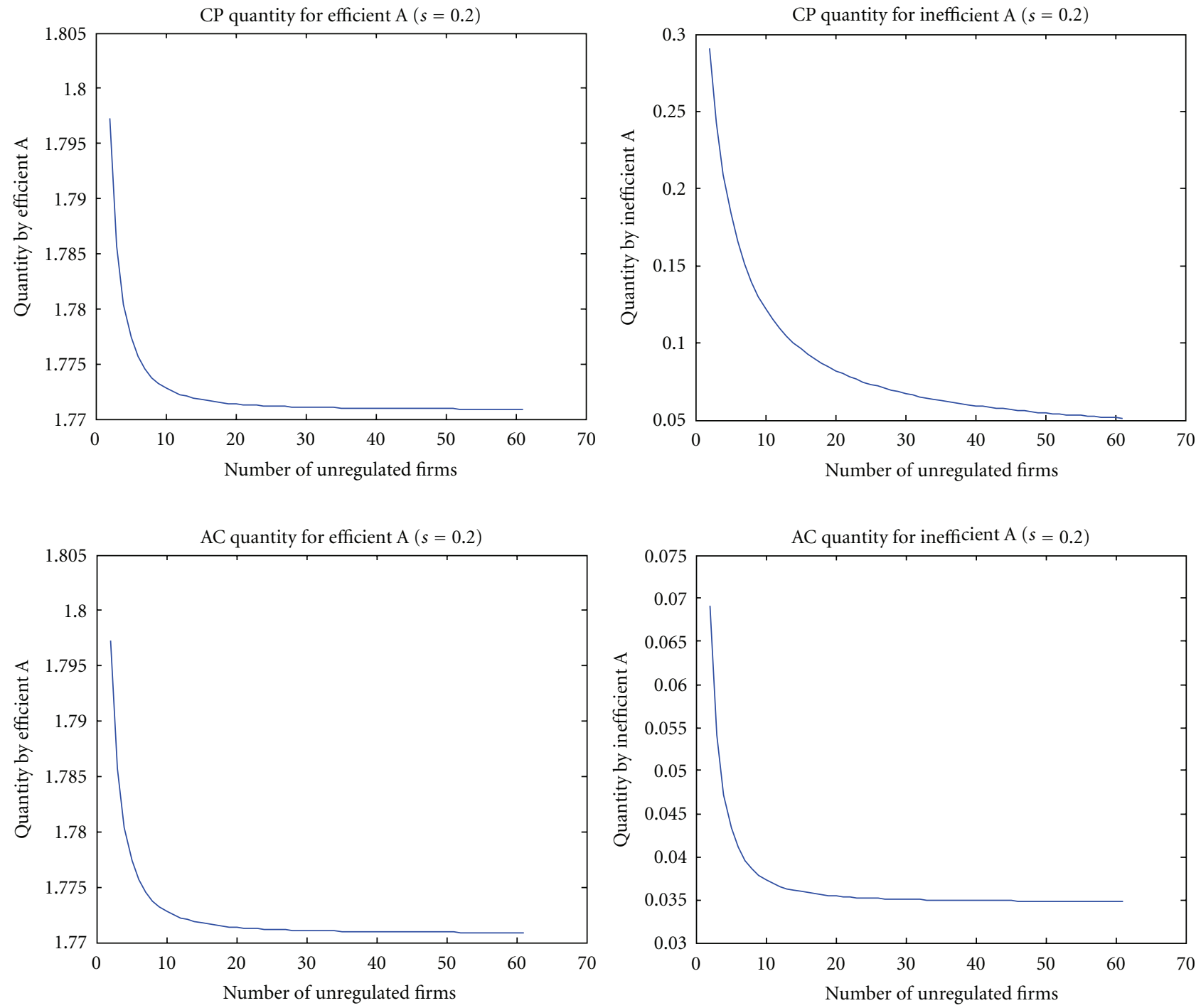

FIgURE 1

From the previous graph we can draw the same conclusions made previously for the number of unregulated firms. The Welfare differences are always positive, which means that the collusion-allowing equilibrium leads to greater social welfare. This Welfare difference increases with the degree of substitutability, which means that as the products are seen as close substitutes the two markets also seem closer and the weight that $F^{A}$ has in the total output and in social welfare is much smaller. Additionally, by allowing collusion, the unregulated firms are able to decrease the information asymmetry in the economy, also decreasing the bias it will have on the Welfare. Once again, we can also notice that the level of Welfare difference increases with the number of unregulated firms.

Finally, it is also worth mentioning that such analysis still holds when the range of values for the unregulated firms' marginal costs increases, creating new unregulated firms that are more efficient than the efficient $F^{A}$ and other unregulated firms that are more inefficient than the inefficient regulated firm (Results and diagrams are in Appendix E.) The only difference lies on the level of values for the Welfare differences, which are still increasing and strictly positive but at even greater values, meaning that with increasing marginal cost differences across firms, not only the regulator does not need to impose costs on society by creating incentives to extract marginal cost information from $F^{A}$, but also the risk that an effective collusion might appear will be much lower.

5.5. The Importance of Consumer Surplus Differences. From the previous section, a somewhat paradoxal result was raised: it is always Welfare improving to allow collusion between the regulated firm and the unregulated firms. Aubert and Pouyet [13] have already stated the failure of the collusion-proofness for some values of the substitutability degree, which was reinforced in this paper by admitting a new dimension to the model (an exogenous fixed number of unregulated firms). The reasons behind such conclusion were also already 

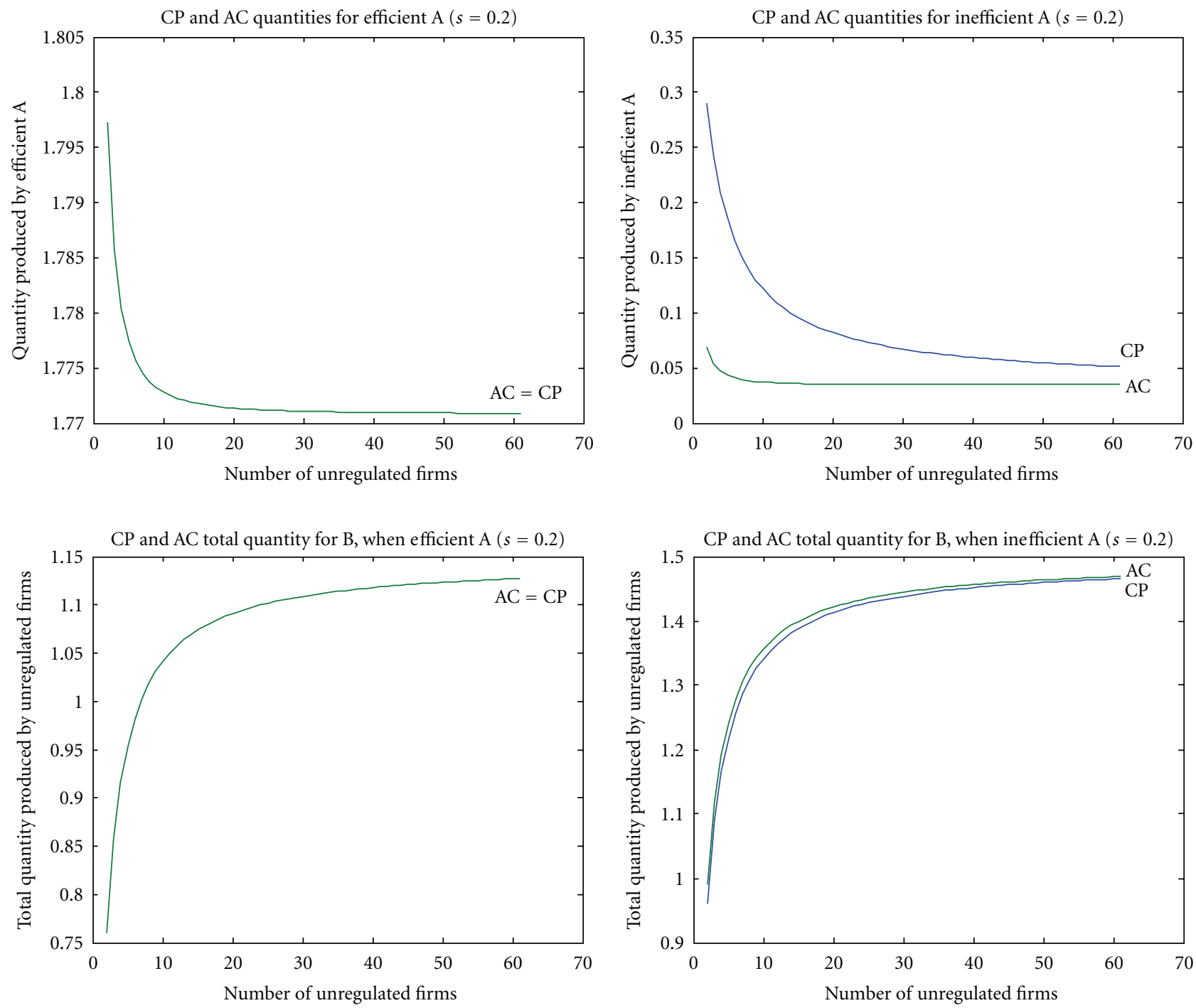

FIgURe 2

stated. In an incomplete regulation framework, the regulator has no control over most of the firms, and therefore he cannot replicate through contracts all the possible outcomes. Hence, it will be socially less costly to allow the unregulated firms, through side-payments, to extract $F^{A}$ 's information regarding her true marginal costs. In the light of such evidence, why do regulators still not allow collusion? The answer to this question may be found if we look only at the Consumer Surplus differences instead of social welfare differences (Figure 6).

The first and most important conclusion drawn from these diagrams is that active collusion will always lead to lower Consumer Surplus values. By allowing collusion, the aggregate quantity produced by both the regulated and unregulated firms will be lower (see the graphs in Section 5.3) and therefore the prices will be higher, which together will harm consumers. However, the number of unregulated firms and the substitutability degree will have opposite effects on the consumer surplus. As the number of unregulated firms increases, the consumer surplus differences will decrease, while such differences will increase with the substitutability degree. Once again both results can be explained by the differences on the aggregate quantity produced. When the number of unregulated firms increases, there is a convergence between the levels of quantities produced and increases in the substitutability degree will lead to greater differences between the collusion-allowing and the collusion-proof outcomes.

Hence, a new and important conclusion rises from this analysis: social welfare differences will depend heavily on the relative weight that the regulator allocates to Consumer Surplus on the social welfare function, also pointed out by Biglaiser and $\mathrm{Ma}$ [12].

\section{Conclusion}

The main purpose of this paper was to add a new dimension to the model put forth by Aubert and Pouyet [13], by 

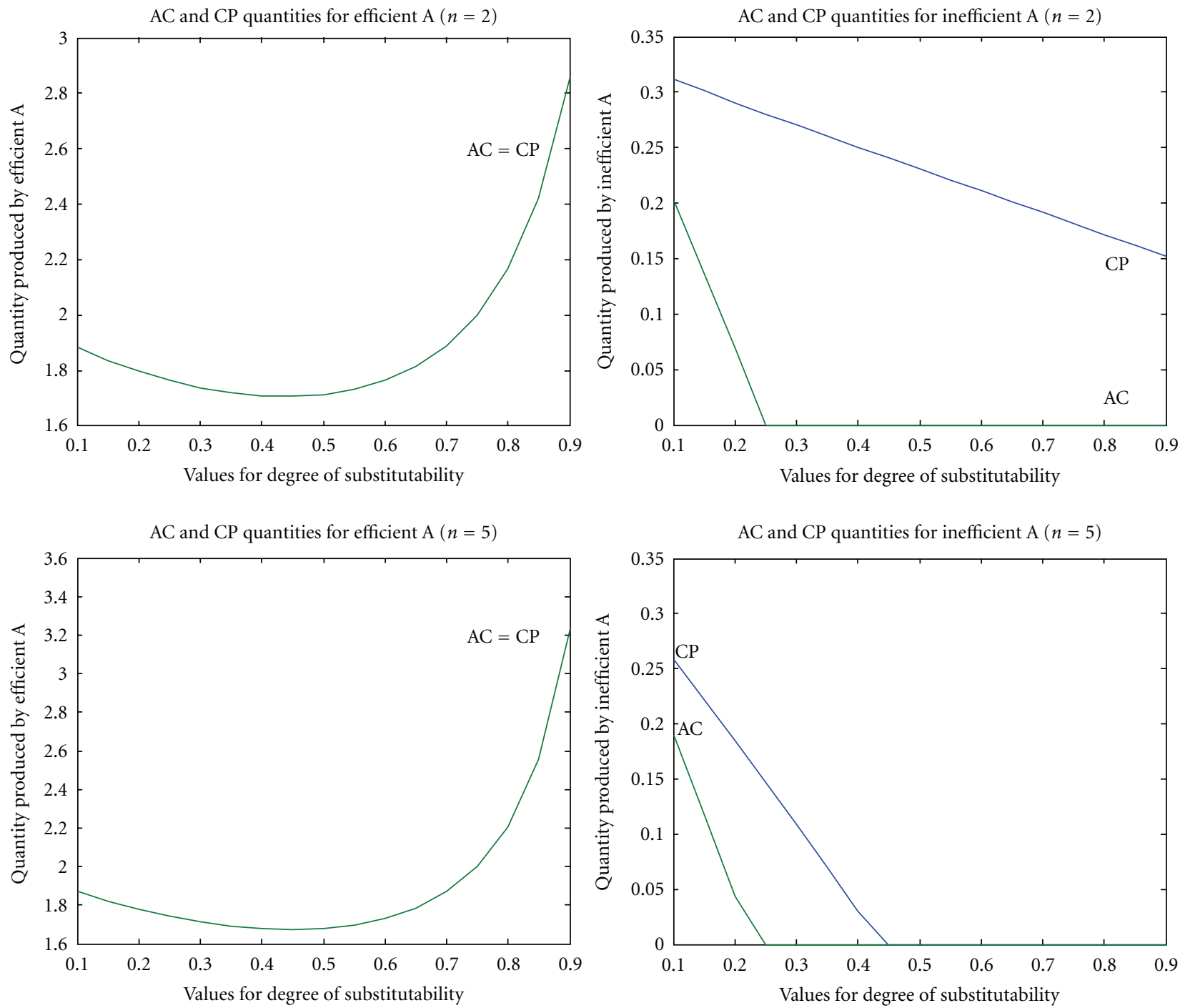

FIgURE 3

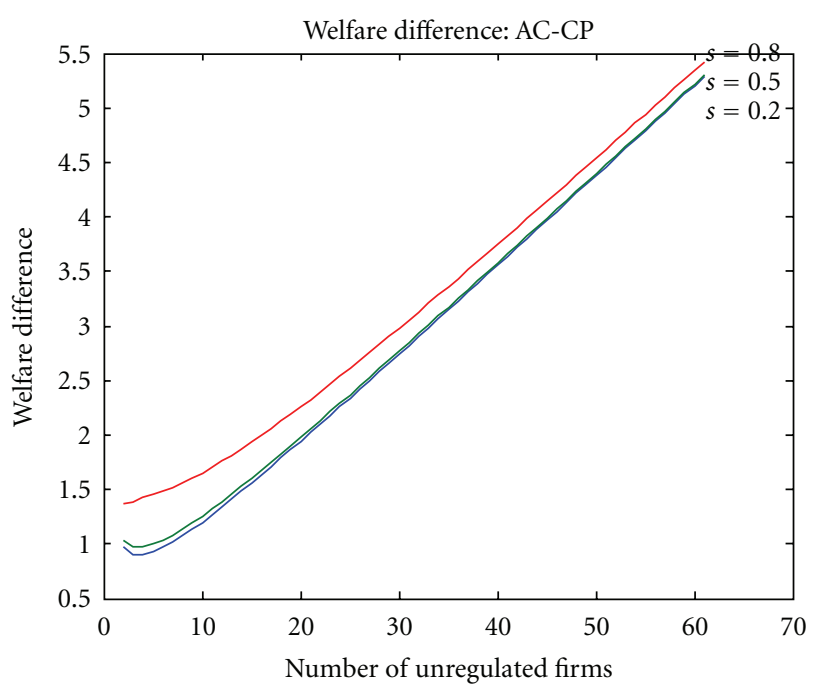

FIGURE 4

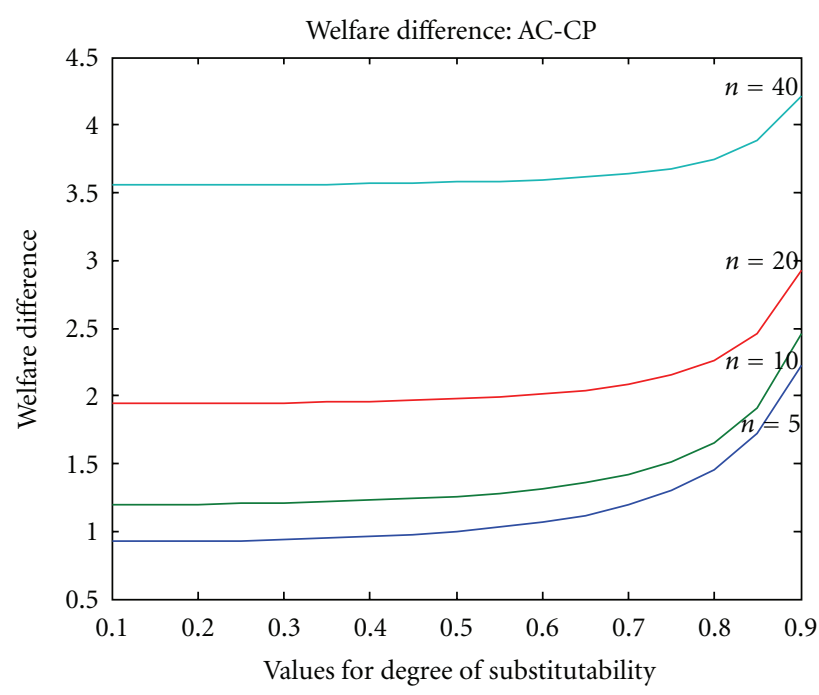

FIgURe 5 

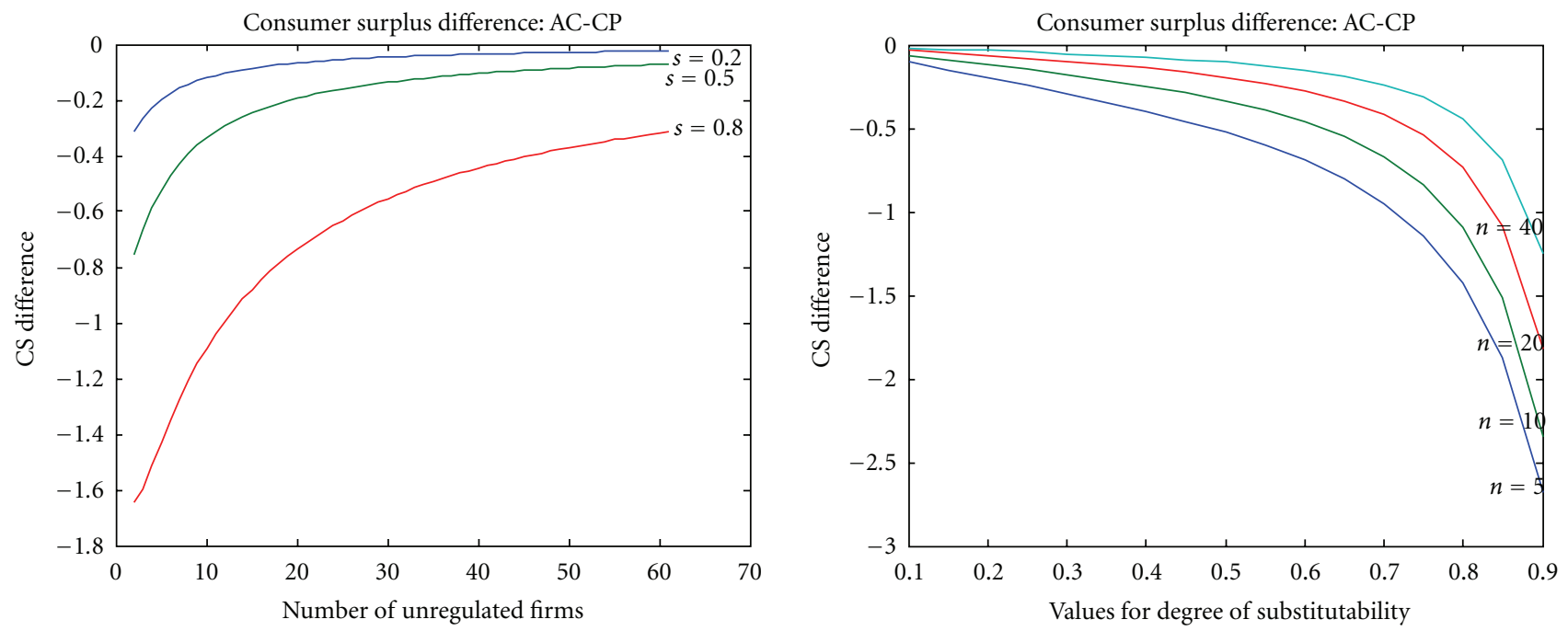

Figure 6

introducing an exogenous number of unregulated firms in the market. This extra dimension was also able to produce a powerful argument against collusion even in situation when apparently it would be Welfare improving to allow it.

Aubert and Pouyet [13] proposed a model of incomplete regulation in a duopoly with asymmetric information regarding the costs of the regulated firm, in a context of product differentiation. The main conclusion of that paper was the failure of the collusion-proofness principle, which stated that higher social welfare would be attained if the regulator designed a contract with the regulated firm that prevented any form of collusion between the two of them. This conclusion was also supported by Tirole [16], Itoh [9], Lawarrëe and Shin [10], and Shin [11].

The model presented in this paper represents an extension, where the regulated firm faces competition from $n$ unregulated firms and analyses the robustness of the conclusions drawn by Aubert and Pouyet [13]. Although this new dimension leads to a much more mathematically demanding model, it has reinforced the failure of the collusion-proofness principle. As the number of unregulated firms increases, the Welfare difference between the collusion-allowing and the collusion-proof equilibriums is always positive and increasing. Such result may be justified by the decreasing weight of the regulated firm in the aggregated market and on the social welfare function and by the ability that the unregulated firms have to extract truthful information regarding the costs of the regulated firm. This result still holds when we consider the Welfare difference for different levels of the substitutability degree. Nevertheless, by analysing the Consumer Surplus differences, we were able to find the most powerful argument for the regulator to still not allow collusion. The results presented earlier depend heavily on the relative weight allocated to the Consumer Surplus in the social welfare function. This new fact brings a new perspective on the results reached by Aubert and Pouyet [13] and on the literature that stated the advantages of allowing collusion.

However there is still some room for further research, by adding more dimensions to the present model. First and foremost, we could start by analysing the role of the weight of the Consumer Surplus in the social welfare function in the simpler framework of only two firms of Aubert and Pouyet [13]. Secondly, we can also assess what would happen if we had again only two firms (one regulated and the other unregulated) and both of them had marginal costs which were private information. Another way of improving the model is to introduce endogeneity in the number of unregulated firms operating in the market, by introducing entry and exit dynamics. Additionally, we could also introduce the possibility of collusion between the regulated firm and only some of the unregulated firms in the market.

\section{Appendices}

\section{A. Incomplete Regulation and Complete Information}

The problem for the regulator when he cannot influence any of the firms in market $\mathrm{B}$, but all that the marginal costs are publicly known will be

$$
\begin{gathered}
\max _{q^{a}} W=G S\left(q^{a}, Q^{b}\right)-\theta^{a} q^{a}-P^{b}\left(q^{a}, Q^{b}\right) Q^{b}-\pi^{a} \\
\text { s.t. } \quad \pi^{a}=0,
\end{gathered}
$$

and therefore, the first-order condition will be

$$
\frac{\partial W}{\partial q^{a}}=\frac{\partial W}{\partial q^{a}}+\frac{\partial W}{\partial Q^{b}} \times \frac{\partial Q^{b}}{\partial q^{a}}=0
$$


which entails the following solution:

$$
\begin{aligned}
q_{*}^{a}\left(\theta^{a}\right)= & \frac{1}{(n+1)^{2}-(n s)^{2}} \\
& \times\left[(n+1)^{2}\left(d^{a}-\theta^{a}\right)-n s\left(n d^{b}-\sum_{i=1}^{n} \theta_{i}^{b}\right)\right] .
\end{aligned}
$$

\section{B. Proof for the Existence of a Stake for Collusion}

There are incentives for collusion since the unregulated firms are better off, by product substitutability, if the firm $F^{A}$ produces the quantity corresponding to her inefficient type.

Using the aggregate reaction function for the firms in market $\mathrm{B}$, and by plugging the equilibrium quantities for $F^{A}$, we get that

$$
\overline{Q^{b}}-\underline{Q^{b}}=\frac{n s(n+1)}{(n+1)^{2}-(n s)^{2}}\left(1+\frac{p}{\bar{p}}\right) \Delta \theta>0,
$$

which proves that the aggregate quantity produced by the firms in market B is greater when the firm $F^{A}$ is inefficient. Substituting both $Q^{b}$ and $q^{a}$, we get the same conclusion for an individual firm $F_{j}^{b}$, for any $j=1, \ldots, n$ :

$$
\overline{q_{j}^{b}}-\underline{q_{j}^{b}}=\frac{s(n+1)}{(n+1)^{2}-(n s)^{2}}\left(1+\frac{\underline{p}}{\bar{p}}\right) \Delta \theta>0,
$$

and substituting this into the individual's profit function for any firm in the unregulated market, we have that

$$
\begin{aligned}
\overline{\pi_{j}^{b}}-\underline{\pi_{j}^{b}}= & \left(d^{b}-\overline{Q^{b}}-s \overline{q^{a}}-\theta_{j}^{b}\right) \overline{q_{j}^{b}} \\
& -\left(d^{b}-\underline{Q^{b}}-s \underline{q^{a}}-\theta_{j}^{b}\right) \underline{q_{j}^{b}} \\
= & \left(d^{b}-\overline{Q^{b}}-s \overline{q^{a}}-\theta_{j}^{b}\right)\left(\overline{q_{j}^{b}}-q_{j}^{b}\right) \\
& +\left[\underline{Q^{b}}-\overline{Q^{b}}+s\left(\underline{q^{a}}-\overline{q^{a}}\right)\right] \underline{q_{j}^{b}}>0 .
\end{aligned}
$$

Hence, each firm in market B will have higher profits if firm $F^{A}$ is inefficient, and therefore there is a stake for collusion.

\section{Collusion-Proof Contracts}

Collusion-Proofness Constraints. As stated before, the collusion-proof contracts are the ones yielding a passive response from the coalition. Since we have a sequence of decisions about the collusion and about the regulatory contract, the first step is to solve the mediator's problem presented earlier.

Let us denote by $v^{k}\left(\theta^{a}\right)$ the multiplier of the coalition participation constraint for the firm $k=a, b$ and by $\delta^{a}\left(\theta^{a}\right)$ the multiplier of the coalition incentive compatibility constraint for each of the types of $F^{A}$. Solving that problem with respect to the side-payments, it yields two first-order conditions that can be combined to obtain a relationship between those multipliers:

$$
v^{a}(\underline{\theta})-v_{i}^{b}(\underline{\theta})=v_{i}^{b}(\bar{\theta})-v^{a}(\bar{\theta}), \quad \forall i=1, \ldots, n .
$$

By plugging the above expression into the mediator's problem, it is possible to separate the collusion problem with respect to reports into two parts:

$$
\begin{aligned}
\max _{\left\{\theta_{m}^{a}(\underline{\theta})\right\}}\left\{\left[\underline{p}+\delta^{a}(\bar{\theta})+v^{b}(\underline{\theta})\right] \pi^{a}\left(\theta_{m}^{a}(\underline{\theta}), \underline{\theta}\right)\right. \\
\left.-\delta^{a}(\bar{\theta}) \pi^{a}\left(\theta_{m}^{a}(\underline{\theta}), \bar{\theta}\right)+\left[\underline{p}+v^{b}(\underline{\theta})\right] \sum_{i=1}^{n} \pi_{i}^{b}\left(\theta_{m}^{a}(\underline{\theta})\right)\right\} \\
\max _{\left\{\theta_{m}^{a}(\bar{\theta})\right\}}\left\{\left[\bar{p}+\delta^{a}(\bar{\theta})+v^{b}(\bar{\theta})\right] \pi^{a}\left(\theta_{m}^{a}(\bar{\theta}), \bar{\theta}\right)\right. \\
\left.-\delta^{a}(\underline{\theta}) \pi^{a}\left(\theta_{m}^{a}(\bar{\theta}), \underline{\theta}\right)+\left[\bar{p}+v^{b}(\bar{\theta})\right] \sum_{i=1}^{n} \pi_{i}^{b}\left(\theta_{m}^{a}(\bar{\theta})\right)\right\} .
\end{aligned}
$$

To ensure collusion-proofness, the regulator must offer a contract such that it always leads to a truth-telling decision from the collusion problem, meaning that $\theta_{m}^{a}(\underline{\theta})=\underline{\theta}$ and $\theta_{m}^{a}(\bar{\theta})=\bar{\theta}$ have to maximize the two objective functions stated above.

Therefore, the two collusion-proofness constraints can be written as

$$
\begin{aligned}
\pi^{a}(\underline{\theta}, \underline{\theta}) \geq & \pi^{a}(\bar{\theta}, \bar{\theta})+\Delta \theta \overline{q^{a}}+\sum_{i=1}^{n} \Delta \pi_{i}^{b} \\
& -\frac{\delta^{a}(\bar{\theta})}{\underline{p}+v^{b}(\underline{\theta})}\left(\underline{q^{a}}-\overline{q^{a}}\right) \Delta \theta \\
\pi^{a}(\bar{\theta}, \bar{\theta}) \geq & \pi^{a}(\underline{\theta}, \underline{\theta})-\Delta \theta \underline{q^{a}}-\sum_{i=1}^{n} \Delta \pi_{i}^{b} \\
& -\frac{\delta^{a}(\underline{\theta})}{\bar{p}+v^{b}(\bar{\theta})}\left(\underline{q^{a}}-\overline{q^{a}}\right) \Delta \theta .
\end{aligned}
$$

For the time being, we will only consider the first collusion-proofness constraint, related to the efficient $F^{A}$, and check afterwards if the last one will always be satisfied. We should also notice that if the regulator induces a truthful report, none of the unregulated firms is willing to pay any side-payment to $F^{A}$, meaning that $b_{i}(\bar{\theta})=b_{i}(\underline{\theta})=0, \quad \forall i=$ $1, \ldots, n$ and that the incentive compatibility constraint for the inefficient $F^{A}$ can be rewritten as $\pi^{a}(\bar{\theta}, \bar{\theta}) \geq \pi^{a}(\underline{\theta}, \underline{\theta})-$ $\Delta \theta q^{a}$, which is exactly the same constraint as the incentive compatibility constraint for an inefficient $F^{A}$ in the regulator's problem. Hence, if this constraint is not binding in the regulator's problem for the best collusion-proof contract, the multiplier $\delta^{a}(\bar{\theta})$ equals to zero, and the collusion-proof constraint for the efficient $F^{A}$ is the same as with perfect information within the coalition: $\pi^{a}(\underline{\theta}, \underline{\theta}) \geq \pi^{a}(\bar{\theta}, \bar{\theta})+\Delta \theta \overline{q^{a}}+$ $\sum_{i=1}^{n} \Delta \pi_{i}^{b}$

The Best Separating Collusion-Proof Contract. We start by assuming that $\delta^{a}(\bar{\theta})$ is not equal to zero, which means that the incentive compatibility constraint for the inefficient $F^{A}$ is not binding in the regulator's problem. Hence the collusionproofness constraint is more stringent than the incentive 
compatibility constraint, and the only binding conditions for the regulator's problem are the participation constraint for the inefficient $F^{A}$ and the collusion-proofness constraint for the efficient $F^{A}$. We just need to check that the equilibrium quantities will satisfy the incentive compatibility constraint for the inefficient $F^{A}$, that is, $q^{a} \geq \overline{q^{a}}$. Knowing that $\pi_{i}^{b}=\left(q_{i}^{b}\right)^{2}$, we get that

$$
\begin{aligned}
\sum_{i=1}^{n} \Delta \pi_{i}^{b}= & \frac{n s}{(n+1)^{2}}\left(\underline{q^{a}}-\overline{q^{a}}\right)\left[2\left(d^{b}+\sum_{i=1}^{n} \theta_{i}^{b}\right)-s\left(\underline{q^{a}}+\overline{q^{a}}\right)\right] \\
& -\frac{2 s}{n+1}\left(\underline{q^{a}}-\overline{q^{a}}\right) \sum_{i=1}^{n} \theta_{i}^{b} .
\end{aligned}
$$

Plugging this expression into the binding constraints we have that

$$
\begin{aligned}
\pi^{a}(\bar{\theta})=0 & \\
\pi^{a}(\underline{\theta})= & \Delta \theta \overline{q^{a}}+\frac{n s}{(n+1)^{2}}\left(\underline{q^{a}}-\overline{q^{a}}\right) \\
\times & {\left[2\left(d^{b}+\sum_{i=1}^{n} \theta_{i}^{b}\right)-s\left(\underline{q^{a}}+\overline{q^{a}}\right)\right]-\frac{2 s}{n+1} } \\
& \times\left(\underline{q^{a}}-\overline{q^{a}}\right) \sum_{i=1}^{n} \theta_{i}^{b}-\frac{\delta^{a}(\bar{\theta})}{\underline{p}+v^{b}(\underline{\theta})}\left(\underline{q^{a}}-\overline{q^{a}}\right) \Delta \theta .
\end{aligned}
$$

Substituting these conditions into the regulator's problem and rearranging the first-order conditions, we obtain the best collusion-proof regulated quantities:

$$
\begin{aligned}
\underline{q_{c p}^{a}}= & \frac{1}{(n+1)^{2}-n s^{2}(2+n)} \\
\times & {\left[(n+1)^{2}\left(d^{a}-\underline{\theta}+\frac{\delta^{a}(\bar{\theta})}{\underline{p}+v^{b}(\underline{\theta})} \Delta \theta\right)\right.} \\
& \left.-(2+n) s\left(n d^{b}-\sum_{i=1}^{n} \theta_{i}^{b}\right)\right], \\
q_{c p}^{a}= & \frac{1}{(n+1)^{2}-n s^{2}(n-2(\underline{p} / \bar{p}))} \\
\times & {\left[\begin{array}{l}
(n+1)^{2}\left(d^{a}-\bar{\theta}-\frac{p}{\bar{p}}\left(1+\frac{\delta^{a}(\bar{\theta})}{p+v^{b}(\underline{\theta})}\right) \Delta \theta\right) \\
\left.-s\left(n-2 \frac{\bar{p}}{\bar{p}}\right)\left(n d^{b}-\sum_{i=1}^{n} \theta_{i}^{b}\right)\right] .
\end{array}\right.}
\end{aligned}
$$

To check if the incentive compatibility constraint for the inefficient $F^{A}$ is satisfied, we need to prove that $\underline{q_{c p}^{a}} \geq \overline{q_{c p}^{a}}$; hence,

$$
\begin{aligned}
& \underline{q_{c p}^{a}}-\overline{q_{c p}^{a}} \\
& =\frac{(n+1)^{2}}{\bar{p}\left[(n+1)^{2}-n s^{2}(2+n)\right]\left[(n+1)^{2}-n s^{2}(n-2(\underline{p} / \bar{p}))\right]} \\
& \quad \times\left\{\begin{array}{c}
2 n s^{2}\left(d^{a}-\underline{\theta}\right)+\left((n+1)^{2}-n s^{2}(2+n)\right) \Delta \theta+ \\
\left.\left((n+1)^{2}-n s^{2}\right) \frac{\delta^{a}(\bar{\theta})}{\underline{p}+v^{b}(\underline{\theta})} \Delta \theta+s \underline{p}\left(\sum_{i=1}^{n} \theta_{i}^{b}-n d^{b}\right)\right\},
\end{array}\right.
\end{aligned}
$$

which is always greater than zero for a small enough $s$. Therefore, if this condition is satisfied, then the incentive compatibility constraint for the best collusion-proof contract for an inefficient $F^{A}$ is not binding, and the relevant collusion-proofness constraint is indeed the same as if the coalition was under complete information. This proves the claim that $\delta^{a}(\bar{\theta})$ is equal to zero and the equilibrium quantities will simplify as in the text. However if this is not satisfied, then the best collusion-proof contract will entail pooling.

The Best Pooling Collusion-Proof Contract. If the previous conditions do not hold, the best collusion-proof contract will entail a pooling equilibrium where the regulator always imposes the same quantity $\overline{q^{a}}$, and therefore no collusion will take place. Hence, the best pooling quantity is the full information for an inefficient firm $F^{A}$.

\section{Application of the Revelation Principle to Collusion-Allowing Contracts}

We will now consider the contracts that do not impose a passive response from the coalition: the collusion-allowing contracts. First, we will prove that the Revelation Principle still applies in the present framework and that we can focus only on direct and truth-telling mechanisms. Finally we will determine the best collusion-allowing contract.

Direct Mechanisms. The revelation principle applies, and we can consider only direct truthful mechanisms $m:\{\underline{\theta}, \bar{\theta}\} \rightarrow$ $\mu \times \mathbb{R}$, mapping a truthful report $\theta^{a}$ by $F^{A}$ into an allocation $\left\{\hat{m}\left(\theta^{a}\right), b\left(\theta^{a}\right)\right\}$. For simplicity, let us denote $\hat{m}(\bar{\theta}) \equiv \bar{m}$ and $\hat{m}(\underline{\theta}) \equiv \underline{m}$. The mediator's problem can then be written as

$$
\begin{array}{rc}
\max _{\left\{\hat{m}\left(\theta^{a}\right), b_{i}\left(\theta^{a}\right)\right\}_{i=1}^{n}} & E_{\theta^{a}}\left[\pi^{a}\left(\hat{m}\left(\theta^{a}\right), b\left(\theta^{a}\right)\right)+\sum_{i=1}^{n} \pi_{i}^{b}\left(\hat{m}\left(\theta^{a}\right)\right)\right] \\
\text { s.t. } & \pi^{a}(\underline{m}, \underline{\theta})+B(\underline{\theta}) \geq \pi^{a}\left(m^{n c}(\underline{\theta}), \underline{\theta}\right) \\
& \text { PC for efficient A } \\
& \pi^{a}(\bar{m}, \bar{\theta})+B(\bar{\theta}) \geq \pi^{a}\left(m^{n c}(\bar{\theta}), \bar{\theta}\right) \\
\text { PC for inefficient A }
\end{array}
$$



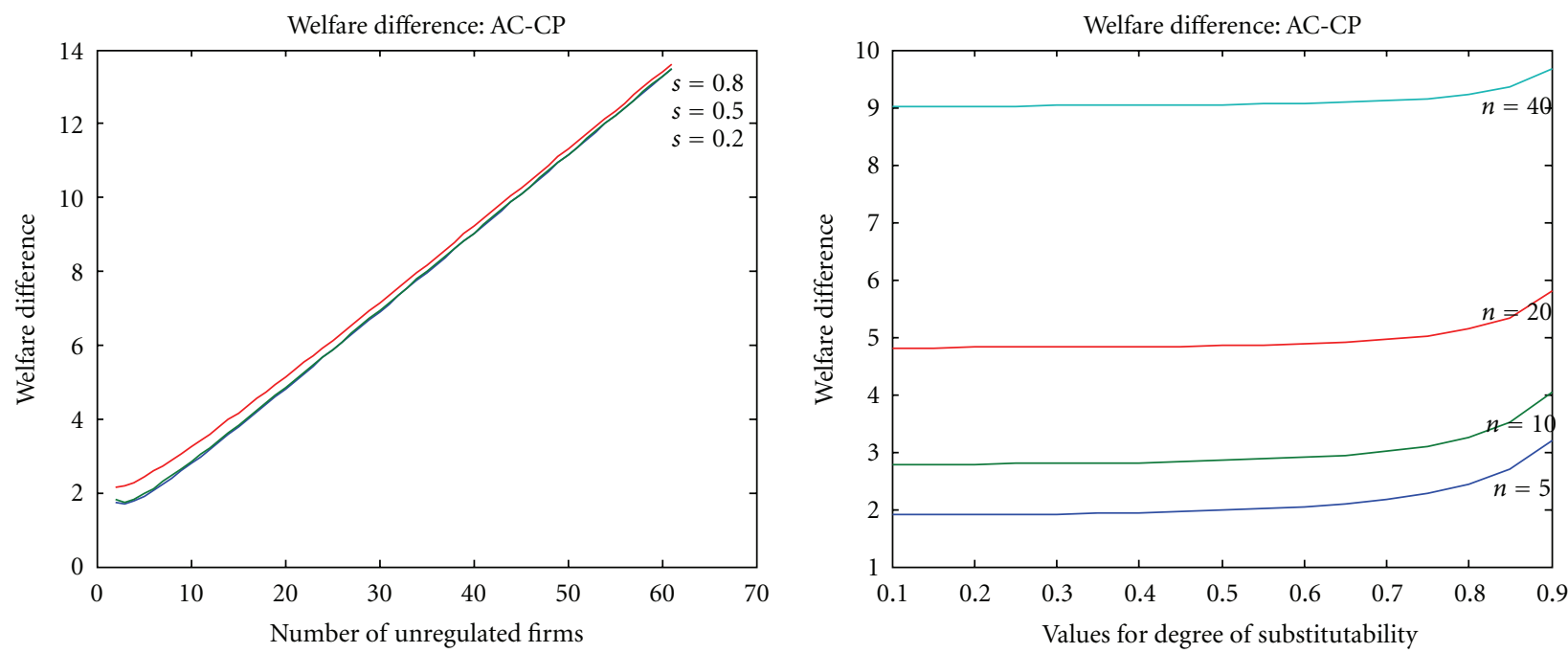

FIgURE 7

$$
\begin{gathered}
\pi^{a}(\underline{m}, \underline{\theta})+B(\underline{\theta}) \geq \pi^{a}(\bar{m}, \underline{\theta})+B(\bar{\theta}) \\
\text { ICC for efficient A } \\
\pi^{a}(\bar{m}, \bar{\theta})+B(\bar{\theta}) \geq \pi^{a}(\underline{m}, \bar{\theta})+B(\underline{\theta}) \\
\text { ICC for inefficient A } \\
\pi_{i}^{b}(\underline{m})-b_{i}(\underline{\theta}) \geq \pi_{i}^{b}\left(m^{n c}(\underline{\theta})\right) \\
\text { PC for each firm in B } \\
\pi_{i}^{b}(\bar{m})-b_{i}(\bar{m}) \geq \pi_{i}^{b}\left(m^{n c}(\bar{\theta})\right) \\
\text { PC for each firm in B. }
\end{gathered}
$$

The next definition will allow us to distinguish clearly the incentive constraints coming from the mediator's problem from the ones coming from the regulator's problem.

Definition 1. A message response $m($.$) that associates some$ messages $\underline{m}$ and $\bar{m}$ in $\mu$ to $\underline{\theta}$ and $\bar{\theta}$, respectively, is said to be $C$-incentive feasible if and only if there exists a couple $\left\{b_{i}(\underline{\theta}), b_{i}(\bar{\theta})\right\} \in \mathbb{R}^{2 n}$ such that all the constraints of the mediator's problem are simultaneously satisfied for $\left\{\underline{m}, \bar{m}, b_{i}(\underline{\theta}), b_{i}(\bar{\theta})\right\}_{i=1}^{n}$.

Let us denote by $\vartheta$ a subset of $\mu \times \mu$ as the set of Cincentive feasible message responses and by $\left\{\underline{m^{T}}, \overline{m^{T}}\right\}$ the solution of the maximization of the aggregated firms' profits. Then we have that

$$
\begin{array}{r}
\left\{\underline{m^{T}}, \overline{m^{T}}\right\} \in \underset{\{\underline{m}, \bar{m}\} \in \mathcal{9}}{\arg \max } \underline{p}\left[\pi^{a}(\underline{m}, \underline{\theta})+\sum_{i=1}^{n} \pi_{i}^{b}(\underline{m})\right] \\
+\bar{p}\left[\pi^{a}(\bar{m}, \bar{\theta})+\sum_{i=1}^{n} \pi_{i}^{b}(\bar{m})\right] .
\end{array}
$$

Lemma 2. If $\{\underline{m}, \bar{m}\}$ is $C$-incentive feasible given an initial message space $\mu$, then it remains so when the message space is reduced to $\left\{\underline{m^{T}}, \overline{m^{T}}\right\}$.
Proof. All the $2 n+4$ constraints can be satisfied for the restricted message space. Considering the participation constraint for the efficient $F^{A}$, if $\{\underline{m}, \bar{m}\}$ is feasible for $\mu$ then that constraint will be satisfied, and there exists a vector given by $\left\{b_{i}(\underline{\theta})\right\}_{i=1}^{n}$ such that $\pi^{a}(\underline{m}, \underline{\theta})+B(\underline{\theta}) \geq \pi^{a}\left(m^{n c}(\underline{\theta}), \underline{\theta}\right)$. By definition of the noncollusive best response, we have that $\pi^{a}\left(m^{n c}(\underline{\theta}), \underline{\theta}\right) \geq \max \left\{\pi^{a}(\underline{m}, \underline{\theta}), \pi^{a}(\bar{m}, \underline{\theta})\right\}$. Hence, the participation constraint for the efficient $F^{A}$ is also satisfied when the message space is restricted. There exists a $\left\{b_{i}(\underline{\theta})\right\}_{i=1}^{n}$ such that $\pi^{a}(\underline{m}, \underline{\theta})+B(\underline{\theta}) \geq \max \left\{\pi^{a}(\underline{m}, \underline{\theta}), \pi^{a}(\bar{m}, \underline{\theta})\right\}$. The same reasoning applies for an inefficient $F^{A}$ and for all the firms in market B. The collusive incentive compatibility constraints are unaffected, and all the constraints are therefore satisfied.

Hence, from the regulator's perspective, there is no restriction in offering a message space $\left\{\underline{m^{T}}, \overline{m^{T}}\right\}$ instead of $\mu$ since the pair is incentive feasible and all other messages are never played.

Truthful Mechanisms. Now we need to prove that there is no restriction on focusing on truthful mechanisms. Before we have seen that there are only two possible messages $\{\underline{\theta}, \bar{\theta}\}$. Therefore we have three possible responses from the coalition when it comes to choosing the report to the regulator: either it always announces the same type, independently of $F^{A}$ 's true costs (which can also be implementable through a truthful mechanism) - it may decide to make a different announcement given $F^{A}$ 's type (which directly corresponds to a truthful mechanism) — or the coalition randomizes on the two messages in at least one state of nature. Hence, it is sufficient to prove that no randomization will take place to prove that there is no loss of generality to focus only on truthful mechanisms.

Randomization occurs only if the coalition total profits are identical for both messages in each state of nature, that is,

$$
\pi^{a}\left(\underline{\theta}, \theta^{a}\right)-\pi^{a}\left(\bar{\theta}, \theta^{a}\right)=\sum_{i=1}^{n}\left[\left(\overline{q_{i}^{b}}\right)^{2}-\left(\underline{q_{i}^{b}}\right)^{2}\right], \quad \forall \theta^{a} .
$$



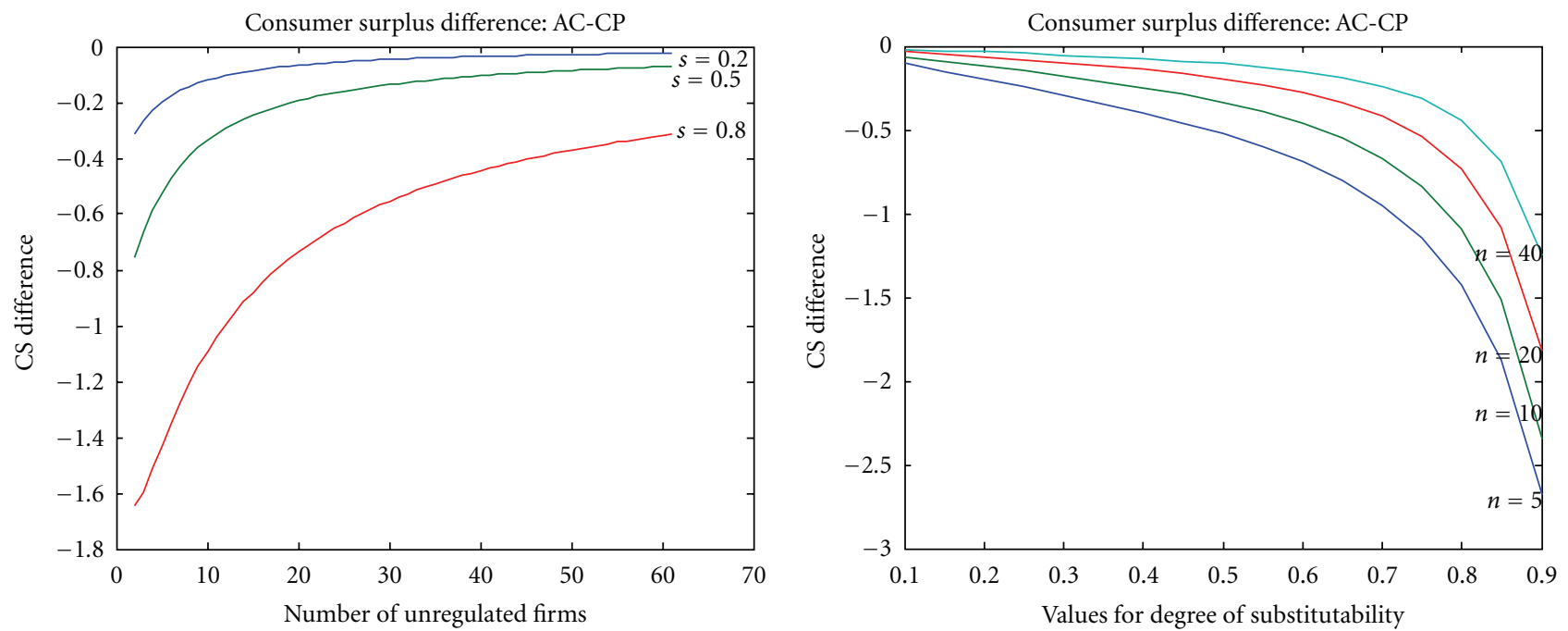

FIgURe 8

Since the regulator's Welfare depends only on $q^{a}$ (since $q_{i}^{b}$ is a function of this quantity) and on the transfers $t^{a}$, we have that only one of the possible two pairs $\left\{q^{a}(\underline{\theta}), t^{a}(\underline{\theta})\right\}$ and $\left\{q^{a}(\bar{\theta}), t^{a}(\bar{\theta})\right\}$ is therefore preferred by the regulator. Hence, if we assume that the coalition is indifferent between the two pairs, the regulator can always offer some additional transfer of $\varepsilon$ (which $s$ approximately zero) for the message that will entail a higher Welfare. Hence, there is no restriction in considering truthful mechanisms.

Lemma 3. There is no loss of generality in considering that only direct and truthful (C-incentive feasible) mechanisms are offered to the coalition.

\section{E. Computational Simulation}

Although comparing equilibrium quantities is not as algebraically demanding as comparing social welfare and Consumer Surplus for the collusion-proof and collusion-allowing equilibriums, we have decided to use graphical representations to illustrate all the comparisons. The graphs result from computational simulation using Matlab.

We have defined the following parameters for firm $F^{A}$ : $d^{a}-\underline{\theta}=2, d^{a}-\bar{\theta}=1$, and $\underline{p}=0.4$. For the unregulated sector a vector of costs was assumed such that for the most efficient firm we had that $d^{b}-\theta_{1}^{b}=2$ and for the most inefficient unregulated firm we had that $d^{b}-\theta_{n}^{b}=1$. This means that the regulated firm $F^{A}$ would always be at the same level of the most efficient or the most inefficient firm of the unregulated sector. Later, the same simulation was done for a wider range of costs for the unregulated sector where $d^{b}-\theta_{1}^{b}=2.3$ and $d^{b}-\theta_{n}^{b}=1.7$.

The simulation was made for a range of values for the substitutability degree such that $0.1 \leq s \leq 0.9$ and for a number of unregulated firms between 2 and 61 . After running the program we ended up with equilibrium quantities matrices of size $(s \times n)$, which fulfilled nonnegativity constraints. We have then used these values to plug into the social welfare and
Consumer Surplus functions to compare them over these two dimensions.

The results for the first vector of marginal costs for the unregulated sector are present in the text. When we consider the wider range of costs for these firms, the graphical results are as follows.

Social Welfare Comparison. From the diagrams above. From Figure 7, we can conclude that a wider range of costs for the unregulated sector would translate into higher levels of social welfare differences. This means that when firm $F^{A}$ is no longer the most efficient nor the least efficient in the market, the collusion-allowing contracts will yield an even better social welfare value, when compared to the collusionproof contracts.

Consumer Surplus Comparison. The above diagrams Figure 8 shows that we still have negative values for the Consumer Surplus difference, which are increasing in number of unregulated firms in the market and decreasing with the substitutability degree, similarly to the results obtained previously for a smaller range of the unregulated firms' marginal costs. Furthermore, the Consumer Surplus differences are approximately the same as before since the two equilibriums are also the same.

\section{References}

[1] D. P. Baron and R. B. Myerson, "Regulating a monopolist with unknown costs," Econometrica, vol. 50, pp. 911-930, 1982.

[2] J.-J. Laffont and J. Tirole, "Using cost information to regulate firms," Journal of Political Economy, vol. 94, pp. 921-937, 1986.

[3] T. R. Lewis and D. Sappington, "Regulating a monopolist with unknown demand," American Economic Review, vol. 78, pp. 986-998, 1988.

[4] B. Caillaud, "Regulation, competition, and asymmetric information," Journal of Economic Theory, vol. 52, no. 1, pp. 87-110, 1990. 
[5] J. J. Laffont and D. Martimort, "Collusion under asymmetric information," Econometrica, vol. 65, no. 4, pp. 875-911, 1997.

[6] J. J. Laffont and D. Martimort, "Mechanism design with collusion and correlation," Econometrica, vol. 68, no. 2, pp. 309 342, 2000.

[7] T. P. Tangerås, “Collusion-proof yardstick competition,” Journal of Public Economics, vol. 83, no. 2, pp. 231-254, 2002.

[8] Y. Che, "Revolving doors and the optimal tolerance for agency collusion," Rand Journal of Economics, vol. 26, pp. 378-397, 1995.

[9] H. Itoh, "Coalitions, incentives, and risk sharing," Journal of Economic Theory, vol. 60, no. 2, pp. 410-427, 1993.

[10] J. Lawarrëe and D. Shin, "Organizational flexibility and cooperative task allocation among agents," Journal of Institutional and Theoretical Economics, vol. 161, no. 4, pp. 621-635, 2005.

[11] D. Shin, "Contracts under wage compression: a case of beneficial collusion," Southern Economic Journal, vol. 74, no. 1, pp. 143-157, 2007.

[12] G. Biglaiser and C. A. Ma, "Regulating a dominant firm: unknown demand and industry structure," The RAND Journal of Economics, vol. 26, no. 1, pp. 1-19, 1995.

[13] C. Aubert and J. Pouyet, "Incomplete regulation, market competition and collusion," Review of Economic Design, vol. 10, no. 2, pp. 113-142, 2006.

[14] J. Green and J.-J. Laffont, "Characterization of satisfactory mechanisms for the revelation of preferences for public goods," Econometrica, vol. 45, pp. 427-435, 1977.

[15] R. Myerson, "Incentive compatibility and the bargaining problem," Econometrica, vol. 47, pp. 61-73, 1979.

[16] J. Tirole, "Hierarchies and bureaucracies: on the role of collusion in organizations," Journal of Law, Economics, and Organization, vol. 2, no. 2, pp. 181-214, 1986. 


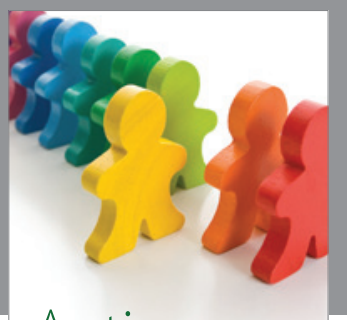

Autism

Research and Treatment
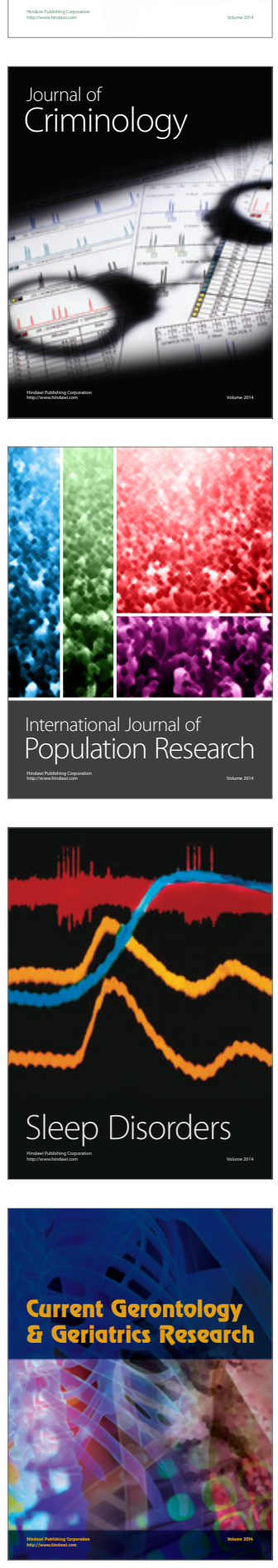
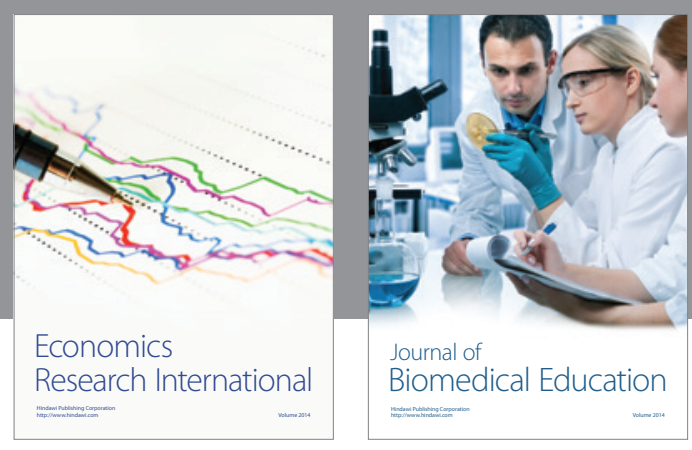

Journal of

Biomedical Education

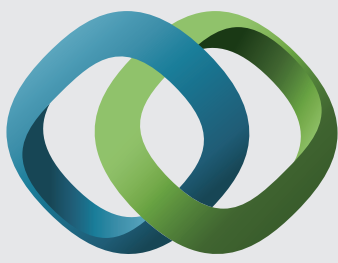

\section{Hindawi}

Submit your manuscripts at

http://www.hindawi.com
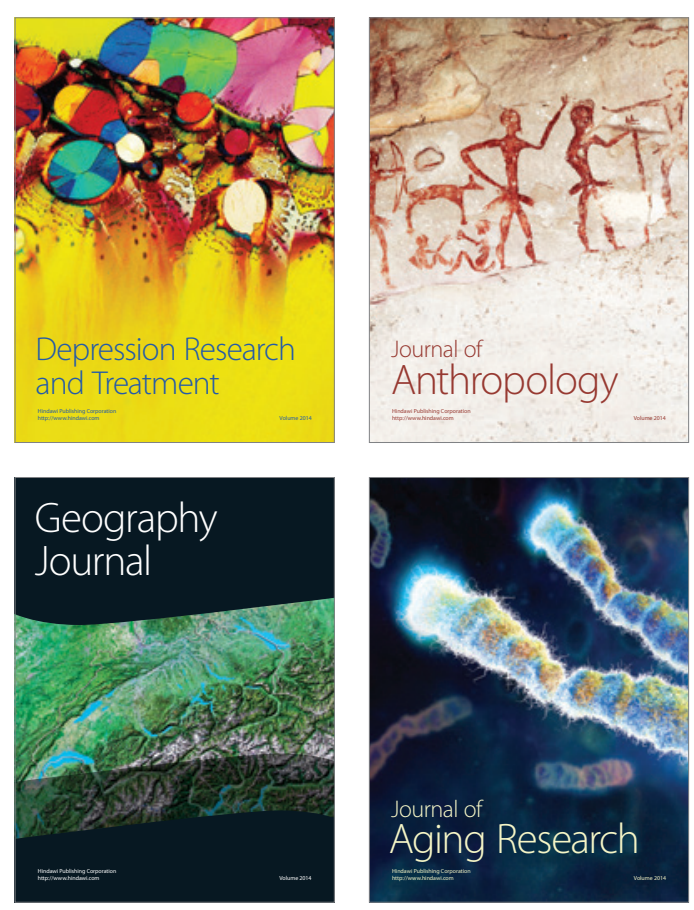

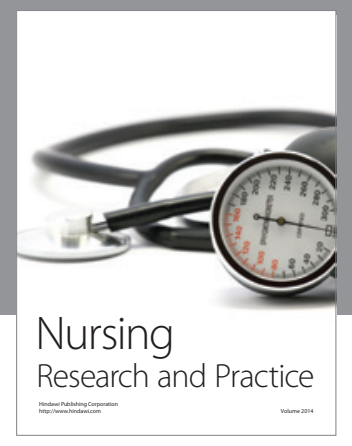

Nursing

Research and Practice

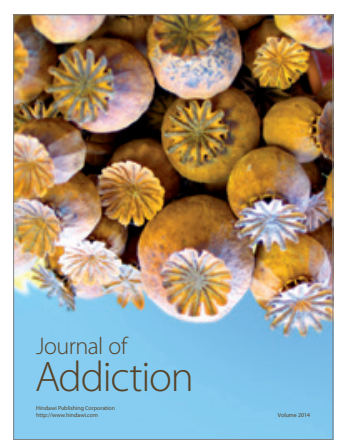

Child Development

Research

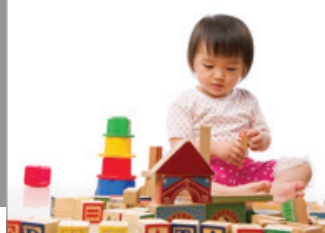

迥
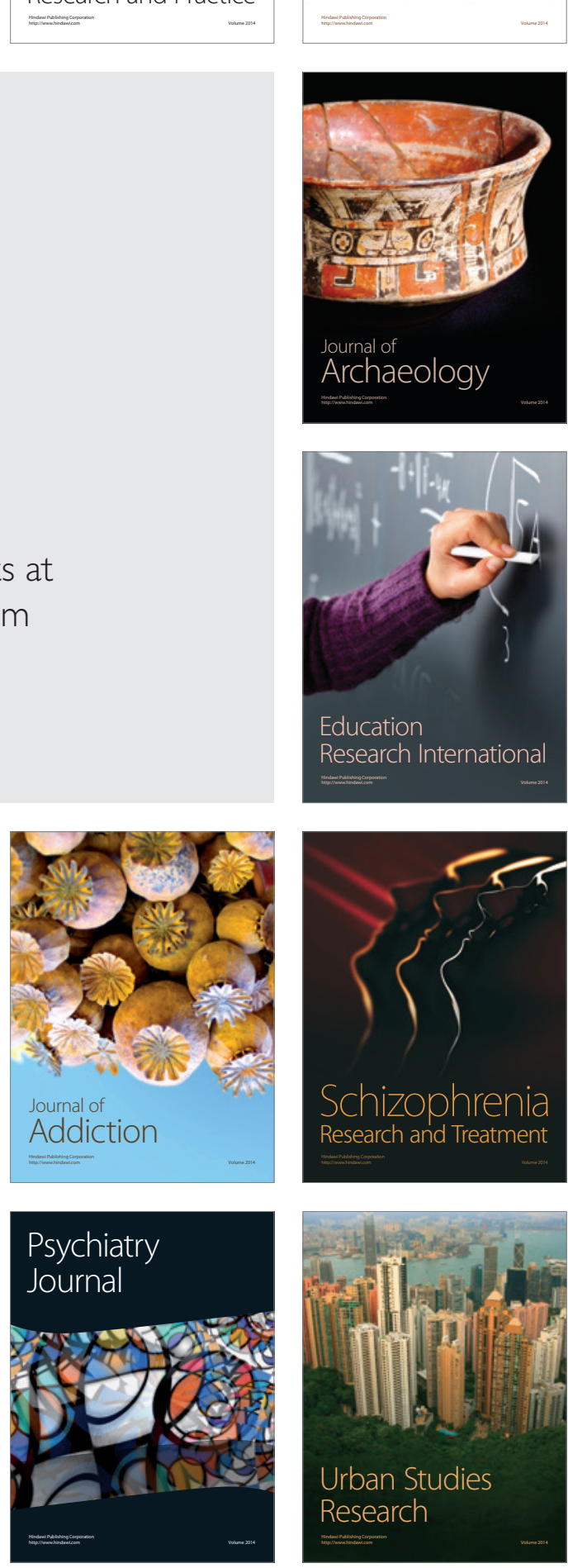